\title{
The effective Shafarevich conjecture for abelian varieties of GL2-type
}

\author{
Rafael von Känel \\ IAS Tsinghua University, Beijing 100084, China; E-mail:rafaelvonkanel@gmail.com.
}

Received: 14 February 2020; Revised: 26 October 2020; Accepted: 11 March 2021

2020 Mathematics Subject Classification: Primary - 11G10 Secondary - 11G50

\begin{abstract}
In this article we establish the effective Shafarevich conjecture for abelian varieties over $\mathbb{Q}$ of $\mathrm{GL}_{2}$-type. The proof combines Faltings' method with Serre's modularity conjecture, isogeny estimates and results from Arakelov theory. Our result opens the way for the effective study of integral points on certain higher dimensional moduli schemes such as, for example, Hilbert modular varieties.
\end{abstract}

\section{Introduction}

In this article we combine Faltings' method with Serre's modularity conjecture to establish the effective Shafarevich conjecture for abelian varieties over $\mathbb{Q}$ of $\mathrm{GL}_{2}$-type.

\subsection{Effective Shafarevich conjecture}

In 1983, Faltings [21] proved the Shafarevich conjecture for (polarised) abelian varieties over number fields. It is known that an effective version of the Shafarevich conjecture would have many striking Diophantine applications. For example, the following effective Shafarevich conjecture $(E S)$ implies the effective Mordell conjecture for curves over number fields (see Section 6). Let $S$ be a nonempty open subscheme of $\operatorname{Spec}(\mathbb{Z})$, and let $g \geq 1$ be a rational integer. We denote by $h_{F}$ the usual stable Faltings height; we recall in Section 2 the definition of this height introduced by Faltings.

Conjecture (ES). There exists an effective constant c, depending only on $S$ and $g$, such that any abelian scheme A over $S$ of relative dimension $g$ satisfies $h_{F}(A) \leq c$.

Coates [11] established (up to a height comparison) the case $g=1$ of this conjecture. He combined a classical reduction of Shafarevich with the theory of logarithmic forms [1]. However, without introducing substantial new ideas, the current state of the art in the theory of logarithmic forms does not allow proving Conjecture $(E S)$ for higher dimensional abelian varieties. In fact, Conjecture $(E S)$ is widely open when $g \geq 2$. We refer to Section 6 for further discussions of this conjecture and related problems.

\section{Abelian schemes of $\mathrm{GL}_{2}$-type.}

Let $A$ be an abelian scheme over $S$ of relative dimension $g$. We say ${ }^{1}$ that $A$ is of $\mathrm{GL}_{2}$-type if $\operatorname{End}(A) \otimes_{\mathbb{Z}} \mathbb{Q}$ contains a number field of degree $g$, where $\operatorname{End}(A)$ denotes the ring of $S$-group scheme morphisms

${ }^{1}$ Here we follow Ribet [59]; some authors use a more restrictive definition (see Section 5).

(C) The Author(s), 2021. Published by Cambridge University Press.. This is an Open Access article, distributed under the terms of the Creative Commons Attribution licence (http://creativecommons.org/licenses/by/4.0/), which permits unrestricted re-use, distribution, and reproduction in any medium, provided the original work is properly cited 
$A \rightarrow A$. For example, any Hilbert-Blumenthal abelian scheme over $S$ is of $\mathrm{GL}_{2}$-type and there is a vast literature on abelian schemes of $\mathrm{GL}_{2}$-type (see Section 5). More generally, we say that $A$ is of product $\mathrm{GL}_{2}$-type if $\operatorname{End}(A) \otimes_{\mathbb{Z}} \mathbb{Q}$ has a commutative semisimple $\mathbb{Q}$-subalgebra of degree $g$. We shall motivate our terminology in (5.1) by showing that $A$ is of product $\mathrm{GL}_{2}$-type if and only if $A$ is isogenous to a product $\prod A_{i}$ of abelian schemes $A_{i}$ over $S$ of $\mathrm{GL}_{2}$-type.

Write $N_{S}=\prod p$ with the product taken over all rational primes $p$ not in $S$; we put here $N_{S}=1$ if $S=\operatorname{Spec}(\mathbb{Z})$. Now we can state our main result (Theorem 6.2).

Theorem A. If $A$ is of product $\mathrm{GL}_{2}$-type, then $h_{F}(A) \leq(3 g)^{144 g} N_{S}^{24}$.

This fully explicit Diophantine inequality establishes Conjecture $(E S)$ for abelian schemes of product $\mathrm{GL}_{2}$-type and it proves new cases of the effective Shafarevich conjecture for curves (see Corollary 6.4). In addition, Theorem A leads to Corollary 6.5, giving new isogeny estimates for abelian schemes over $S$ of product $\mathrm{GL}_{2}$-type: Our isogeny estimates are uniform in the sense that they only depend on $S$ and $g$, which is crucial for Theorem B and for the Diophantine applications in [40]. In Theorem A it is important that we do not make any semistable assumption or assume that $A$ is simple, because these assumptions would be too restrictive for many Diophantine applications of interest.

We next consider the problem of effectively bounding, in terms of $S$ and $g$, the number of isomorphism classes of abelian schemes over $S$ of relative dimension $g$. This problem is still widely open ${ }^{2}$ in the case when $g \geq 2$. However, Theorem A now allows solving the case of abelian schemes of product $\mathrm{GL}_{2}$-type: In Theorem 6.6 we obtain the following quantitative finiteness result for the set $M_{\mathrm{GL}_{2}, g}(S)$ of isomorphism classes of abelian schemes over $S$ of relative dimension $g$ that are of product $\mathrm{GL}_{2}$-type.

Theorem B. The cardinality of $M_{\mathrm{GL}_{2}, g}(S)$ is at most $(14 g)^{(9 g)^{6}} N_{S}^{(18 g)^{4}}$.

We made some effort to obtain an upper bound in Theorem B that is polynomial in terms of $N_{S}$ for each $g \geq 1$. In the important special case $g=1$, Brumer-Silverman [9], Poulakis [53] and Ellenberg, Helfgott and Venkatesh $[18,30]$ already established such polynomial upper bounds: They obtained significantly better exponents of $N_{S}$ by using completely different methods, based on Diophantine approximation or the theory of logarithmic forms; for an overview see the discussions surrounding [38, Prop 6.4]. However, their methods do not allow dealing with abelian varieties of dimension $g \geq 2$, because they all crucially exploit Weierstrass equations for elliptic curves.

\section{Explicit Diophantine applications.}

Theorem A is an effective Diophantine approximation result for $S$-integral points on certain 'spaces' $M_{\mathrm{GL}_{2}, g}$ of arbitrarily large dimension. We now discuss some explicit Diophantine applications. Without introducing new ideas it seems difficult to find applications for rational points on curves of genus at least two, because Paršin's construction [51] (see also Jorgenson-Kramer [34]) usually does not have image in $M_{\mathrm{GL}_{2}, g}$. However, in [38, 42] we showed that there are many other Diophantine problems of interest that can be reduced to the study of $M_{\mathrm{GL}_{2}, g}(S)$ via 'Paršin constructions' given by forgetful maps of moduli schemes. For example, on combining such Paršin constructions with Conjecture $(E S)$ for $M_{\mathrm{GL}_{2}, g}(S)$ with $g=1$, we proved in [38] explicit finiteness results for Diophantine equations inducing integral points on moduli schemes of elliptic curves. Moreover, in the joint work with Matschke [42], we refined the strategy for certain moduli schemes. This led to the construction of new algorithms that allows solving various fundamental Diophantine problems, including $S$-unit, Mordell, cubic Thue, cubic Thue-Mahler and generalised Ramanujan-Nagell equations. To demonstrate the efficiency and utility of the method, we solved large classes of classical equations and we used the resulting data to formulate and motivate new conjectures.

${ }^{2}$ Deligne [14] solved the analogous (but substantially easier) problem for isogeny classes by using and refining certain parts of Faltings' proof of the Shafarevich conjecture for abelian varieties [21]. 
Now, our proof of Conjecture $(E S)$ for $M_{\mathrm{GL}_{2}, g}(S)$ with $g \geq 2$ opens the way for the effective study of some classes of Diophantine equations that appear to be beyond the reach of the known effective methods. For instance, Theorems A and B are the main tools of the joint project with Kret [40, 41]: Therein we combine the results of the present article with suitable Paršin constructions in order to prove explicit upper bounds for the height and the number of $S$-integral points on Hilbert modular varieties. To this end, if $g$ is not too large, say $g \leq 100$, then our explicit height bounds in Theorem A are in fact already sufficiently strong for practical computations when combined with efficient sieves (see [42]) that in practice can deal with huge initial bounds.

\subsection{Principal ideas of the proofs}

We continue our notation. Suppose that $A$ is an abelian scheme over $S$ of relative dimension $g \geq 1$ that is of product $\mathrm{GL}_{2}$-type, with generic fibre $A_{\mathbb{Q}}$. To prove Conjecture $(E S)$ for $A$, we combine Faltings' method [21] with the following tools:

(i) If $A_{\mathbb{Q}}$ is $\mathbb{Q}$-simple, then it is a quotient $J_{1}(N) \rightarrow A_{\mathbb{Q}}$ of the usual modular Jacobian $J_{1}(N)$ of some level $N$. Ribet [59] deduced this statement from Serre's modularity conjecture [43] by using among other things the Tate conjecture for abelian varieties [21].

(ii) Isogeny estimates for abelian varieties over number fields. These estimates were proven by the method of Faltings [21] or by the method of Masser-Wüstholz [46, 47] via the theory of logarithmic forms; see Section 3 for more details.

(iii) Bost's [5] lower bound for $h_{F}$ in terms of the dimension and Javanpeykar's [31] upper bound for the stable Faltings height of Belyi curves in terms of the Belyi degree and the genus. These results are based on Arakelov theory.

Let $N_{A}$ be the conductor of $A$, defined in Subsection 2.2. In the proof we first consider the case when $A_{\mathbb{Q}}$ is $\mathbb{Q}$-simple. A result of Carayol [10] allows controlling the number $N$ in (i). This together with (i)-(iii) leads to an effective bound for $h_{F}(A)$ in terms of $N_{A}$ and $g$ and then in terms of $N_{S}$ and $g$ because $A$ is an abelian scheme over $S$. To reduce the general case to the case when $A_{\mathbb{Q}}$ is $\mathbb{Q}$-simple, we use inter alia Poincaré's reducibility theorem and again the isogeny estimates in (ii). For the proof of Conjecture $(E S)$ for $A$, each of the two methods in (ii) is sufficient. However, to obtain the bound in Theorem A we used in (ii) the isogeny estimates based on the method of Masser-Wüstholz, see Remark 1.

We now describe the principal ideas of our proof of Theorem B. Following Faltings [21] we divide our quantitative finiteness proof for $M_{\mathrm{GL}_{2}, g}(S)$ into the following two parts: (a) finiteness of $M_{\mathrm{GL}_{2}, g}(S)$ up to isogenies and (b) finiteness of each isogeny class of $M_{\mathrm{GL}_{2}, g}(S)$. To prove part (a) we use (i) and we show that any $\mathbb{Q}$-simple 'factor' $A_{i}$ of $A_{\mathbb{Q}}$ is a quotient

$$
J_{1}(v) \rightarrow A_{i}
$$

where $v$ is a rational integer depending only on $S$ and $g$. To show part (b) we combine Theorem A with an estimate of Masser-Wüstholz [46, 47] for the minimal degree of isogenies of abelian varieties that is based on the theory of logarithmic forms. In fact, we use here the recent version of the Masser-Wüstholz estimate, due to Gaudron-Rémond [25].

Moreover, the just described strategy for Theorems A and B gives effective versions of the Shafarevich conjecture for the class of abelian varieties that are quotients of Jacobians $\operatorname{Pic}^{0}(X)$ of Belyi curves $X$ with suitably controlled Belyi degree. However, substantially new ideas are required to generalise Theorems A and B to arbitrary number fields $K$ because (i) is not available for a general $K$. Also, we cannot apply here our reduction to $K=\mathbb{Q}$ used in the proof of Proposition 6.1 because the notion of (product) GL $2^{-}$ type is not stable under Weil restriction. Nevertheless, for certain abelian varieties of interest that are defined over an arbitrary $K$, one can reduce via Weil restriction to Theorems A and B; see, for example, [36, Prop 9.9], which is crucial for explicitly bounding in [41] the height and the number of integral points on coarse Hilbert moduli schemes. 


\section{Remarks.}

1) The theory of logarithmic forms [1] is a powerful tool that is often indispensable for proving effective Diophantine finiteness results. Right at the beginning of Subsection 6.2.2, we give a proof of Conjecture $(E S)$ for abelian schemes $A$ over $S$ of product $\mathrm{GL}_{2}$-type that does not rely on the theory of logarithmic forms. This proof uses in (ii) the isogeny estimates [54] based on Faltings' method. However, using in (ii) the isogeny estimates [25, 46] based on the theory of logarithmic forms leads to the bounds in Theorems A and B, which are at least exponentially better in terms of $N_{S}$ and the relative dimension $g$ of the abelian scheme $A$ over $S$.

2) The present article is the second part of [36]. In the first part, published in [38], we used the ShimuraTaniyama conjecture $[7,66,68]$ to prove Theorems A and B for $g=1$. In particular, in [36, Cor 6.3] we established the effective bound $h_{F}(A) \ll_{\epsilon} N_{S}^{2+\epsilon}$. To obtain this bound, we first reduced to an explicit height-conductor inequality and then we proved such an inequality (proven independently by Murty-Pasten [50]) by using Frey's approach [23] via the modular degree and congruences. This approach gives better bounds for $g=1$, but it is not available for $A \in M_{\mathrm{GL}_{2}, g}(S)$ with $g \geq 2$. In [36], the proof of Theorem B for $g=1$ uses Mazur's uniform isogeny results. Because such uniform results are not available for $g \geq 2$ and because $A \in M_{\mathrm{GL}_{2}, g}(S)$ is not necessarily simple, we had to find other arguments (described above): For each $g$ they lead to a bound in Theorem B that is still polynomial in terms of $N_{S}$ but with a quite large exponent. In general we tried to simplify the form of the explicit bounds appearing in our results. Hence, it would be possible to state our results with explicit bounds that are slightly better. However, bounds of the form $h_{F}(A) \ll_{g} \log N_{S}$ are currently out of reach for any of the above discussed methods, even for $g=1$.

\subsection{Organisation of the article}

\section{Outline.}

In Section 2 we discuss properties of Faltings heights and of the conductor of abelian varieties over number fields. Then in Section 3 we collect results that control the variation of Faltings heights in an isogeny class. In Section 4 we work out explicit estimates for the stable Faltings heights of Jacobian varieties of certain classical modular curves. Then we prove in Section 5 a height-conductor inequality for abelian varieties over $\mathbb{Q}$ of product $\mathrm{GL}_{2}$-type. Finally, in Section 6 we establish the effective Shafarevich conjecture $(E S)$ for abelian schemes of product $\mathrm{GL}_{2}$-type and we deduce some applications. We also added two appendices: First we apply our arguments to prove an asymptotic height-conductor inequality for semistable abelian varieties over $\mathbb{Q}$ with real multiplications, and in the second appendix we simplify the shape of Raynaud's bound [54, Thm 4.4.9].

\section{Notation, conventions and terminology.}

Let $K$ be a number field with ring of integers $\mathcal{O}_{K}$. We identify a nonzero prime ideal of $\mathcal{O}_{K}$ with the corresponding finite place $v$ of $K$ and vice versa. We write $N_{v}$ for the number of elements in the residue field of $v$, we denote by $v(\mathfrak{a})$ the order of $v$ in a fractional ideal $\mathfrak{a}$ of $K$ and we write $v \mid \mathfrak{a}$ (respectively $v \nmid \mathfrak{a})$ if $v(\mathfrak{a}) \neq 0$ (respectively $v(\mathfrak{a})=0$ ). Let $A$ be an abelian variety over $K$. We say that $A$ is semistable if it has semistable reduction at all finite places of $K$.

Let $S$ be an arbitrary scheme. We often identify an affine scheme $S=\operatorname{Spec}(R)$ with the $\operatorname{ring} R$. If $T$ and $Y$ are $S$-schemes, then we denote by $Y(T)=\operatorname{Hom}_{S}(T, Y)$ the set of $S$-scheme morphisms from $T$ to $Y$ and we write $Y_{T}=Y \times_{S} T$ for the base change of $Y$ from $S$ to $T$. Further, if $A$ and $B$ are abelian schemes over $S$, then we denote by $\operatorname{Hom}(A, B)$ the abelian group of $S$-group scheme morphisms from $A$ to $B$ and we write $\operatorname{End}(A)=\operatorname{Hom}(A, A)$ for the endomorphism ring of $A$. Following [4], we say that $S$ is a Dedekind scheme if $S$ is a normal noetherian scheme of dimension 0 or 1 .

By log we mean the principal value of the natural logarithm and we define the maximum of the empty set and the product taken over the empty set as 1 . For any set $M$, we denote by $|M|$ the (possibly infinite) number of distinct elements of $M$. 


\section{Height and conductor of abelian varieties}

Let $K$ be a number field and let $A$ be an abelian variety over $K$. In the first part of this section, we recall the definition of the relative and the stable Faltings height of $A$, and we review fundamental properties of these heights. In the second part, we define the conductor $N_{A}$ of $A$ and we recall useful properties of $N_{A}$.

\subsection{Faltings heights}

We begin to define the relative and stable Faltings height of $A$ following [21, p. 354]. If $A=0$, then we set $h(A)=0$. We now assume that $A$ has positive dimension $g \geq 1$. Let $B$ be the spectrum of the ring of integers $\mathcal{O}_{K}$ of $K$. We denote by $\mathcal{A}$ the Néron model of $A$ over $B$, with zero section $e: B \rightarrow \mathcal{A}$. Let $\Omega^{g}$ be the sheaf of relative differential $g$-forms of $\mathcal{A} / B$. We now metrise the line bundle $\omega=e^{*} \Omega^{g}$ on $B$. For any embedding $\sigma: K \hookrightarrow \mathbb{C}$, we denote by $A^{\sigma}$ the base change of $A$ to $\mathbb{C}$ with respect to $\sigma$. We choose a nonzero global section $\alpha$ of $\omega$. Let $\left\|\alpha_{\sigma}\right\|_{\sigma}$ be the positive real number that satisfies

$$
\left\|\alpha_{\sigma}\right\|_{\sigma}^{2}=\left(\frac{i}{2}\right)^{g} \int_{A^{\sigma}(\mathbb{C})} \alpha_{\sigma} \wedge \overline{\alpha_{\sigma}},
$$

where $\alpha_{\sigma}$ denotes the holomorphic differential form on $A^{\sigma}$ that is induced by $\alpha$. Then the relative Faltings height $h(A)$ of $A$ is the real number defined by

$$
[K: \mathbb{Q}] h(A)=\log \left|\omega / \alpha \mathcal{O}_{K}\right|-\sum \log \left\|\alpha_{\sigma}\right\|_{\sigma}
$$

with the sum taken over all embeddings $\sigma: K \hookrightarrow \mathbb{C}$. The product formula assures that this definition does not depend on the choice of $\alpha$. The relative Faltings height is compatible with products of abelian varieties: If $A^{\prime}$ is an abelian variety over $K$, then $h\left(A \times_{K} A^{\prime}\right)=h(A)+h\left(A^{\prime}\right)$. To see the behaviour of $h$ under base change we take a finite field extension $L$ of $K$. The universal property of Néron models implies

$$
h\left(A_{L}\right) \leq h(A) .
$$

This inequality can be strict and thus the height $h$ is in general not stable under base change. To obtain a stable height we may (see [29]) and do take a finite extension $L^{\prime}$ of $K$ such that $A_{L^{\prime}}$ is semistable. The stable Faltings height $h_{F}(A)$ of $A$ is defined as

$$
h_{F}(A)=h\left(A_{L^{\prime}}\right) .
$$

This definition does not depend on the choice of $L^{\prime}$, because the formation of the identity components of the corresponding semistable Néron models commutes with the induced base change. In particular, inequality (2.1) becomes an equality when $h$ is replaced by $h_{F}$. Further, we define $h_{F}(0)=0$. We shall need an effective lower bound for $h_{F}(A)$ in terms of the dimension $g$ of $A$. An explicit result of Bost [5] gives

$$
-\frac{g}{2} \log \left(2 \pi^{2}\right) \leq h_{F}(A) .
$$

See, for example, [26, Corollaire 8.4] and notice that $h_{F}(A)=h_{B}(A)-\frac{g}{2} \log \pi$ where $h_{B}$ denotes the height, which appears in the statement of [26, Corollaire 8.4].

We shall state several of our results in terms of $h_{F}$ or $h$ and therefore we now briefly discuss important differences between these heights. From (2.1) we deduce that $h_{F}(A) \leq h(A)$. Further, as already observed, the height $h_{F}$ has the advantage over $h$ that it is stable under base change. On the other hand, $h_{F}$ has in general weaker finiteness properties. For instance, there are only finitely many $K$-isomorphism classes of elliptic curves over $K$ of bounded $h$, and $h_{F}$ is bounded on the infinite set given by the $K$-isomorphism classes of elliptic curves of any fixed $j$-invariant in $K$. 
More generally, let $S$ be a connected Dedekind scheme with field of fractions $K$. If $A$ is an abelian scheme over $S$, then we define the stable and relative Faltings height of $A$ by $h_{F}(A)=h_{F}\left(A_{K}\right)$ and $h(A)=h\left(A_{K}\right)$, respectively.

\subsection{Conductor}

We first define the conductor $N_{A}$ of an arbitrary abelian variety $A$ over any number field $K$. Let $v$ be a finite place of $K$. We denote by $f_{v}$ the usual conductor exponent of $A$ at $v$; see, for example, [61, Subsection 2.1] for a definition. The conductor $N_{A}$ of $A$ is defined by

$$
N_{A}=\prod N_{v}^{f_{v}}
$$

with the product taken over all finite places $v$ of $K$. In particular, $f_{v}(0)=0$ and $N_{0}=1$. We now recall some useful properties of $f_{v}$ and $N_{A}$. It holds that $f_{v}=0$ if and only if $A$ has good reduction at $v$. Furthermore, if $A^{\prime}$ is an abelian variety over $K$ that is $K$-isogenous to $A$, then $f_{v}(A)=f_{v}\left(A^{\prime}\right)$ and thus $N_{A}=N_{A^{\prime}}$. Finally, if $A^{\prime}$ is an abelian variety over $K$ and if $C=A \times_{K} A^{\prime}$, then $f_{v}(C)=f_{v}(A)+f_{v}\left(A^{\prime}\right)$ and hence $N_{C}=N_{A} N_{A^{\prime}}$.

We shall need an explicit upper bound for $f_{v}$ in terms of $g=\operatorname{dim}(A)$ and $K$. Brumer-Kramer [8] obtained such a bound by refining earlier work of Serre [62, Subsection 4.9] and of Lockhart-RosenSilverman [45]. To state the main result of [8] we have to introduce some notation. Let $p$ be the residue characteristic of $v$, let $e_{v}=v(p)$ be the ramification index of $v$ and let $n$ be the largest integer that satisfies $n \leq 2 g /(p-1)$. We define $\lambda_{p}(n)=\sum i r_{i} p^{i}$ for $\sum r_{i} p^{i}$ the $p$-adic expansion of $n=\sum r_{i} p^{i}$ with integers $0 \leq r_{i} \leq p-1$. Then [8, Theorem 6.2] gives

$$
f_{v} \leq 2 g+e_{v}\left(p n+(p-1) \lambda_{p}(n)\right) .
$$

Furthermore, the examples in [8] show that (2.4) is best possible in a strong sense. In what follows, we shall always combine (2.4) with the upper bound $\lambda_{p}(n) \leq n\left\lfloor\frac{\log n}{\log p}\right\rfloor$ where for any real number $x$ we write $\lfloor x\rfloor$ for the largest integer at most $x$.

More generally, if $S$ is a connected Dedekind scheme with field of fractions $K$ and if $A$ is an abelian scheme over $S$, then we define the conductor $N_{A}$ of $A$ by $N_{A}=N_{A_{K}}$.

\section{Variation of Faltings heights under isogenies}

In this section, we collect results that control the variation of Faltings heights under isogenies. These results are rather direct consequences of theorems in the literature.

Let $K$ be a number field and let $A$ be an abelian variety over $K$ of dimension $g \geq 1$. We denote by $h_{F}(A)$ the stable Faltings height of $A$ and by $h(A)$ the relative Faltings height of $A$; see Section 2 for the definitions. The results of Faltings [21, Lemma 5] and Raynaud [54, Corollaire 2.1.4] provide that any $K$-isogeny $\varphi: A \rightarrow A^{\prime}$ of abelian varieties over $K$ satisfies

$$
\left|h(A)-h\left(A^{\prime}\right)\right| \leq \frac{1}{2} \log \operatorname{deg}(\varphi)
$$

Let $N_{A}$ be the conductor of $A$ defined in Subsection 2.2, let $D_{K}$ be the absolute value of the discriminant of $K$ over $\mathbb{Q}$ and let $d=[K: \mathbb{Q}]$ be the degree of $K$ over $\mathbb{Q}$.

Lemma 3.1. Suppose $A^{\prime}$ is an abelian variety defined over $K$ that is $K$-isogenous to $A$. Then the following statements hold:

(i) There exists an effective constant $\mu$, depending only on $g, N_{A}, d$ and $D_{K}$, such that

$$
\left|h_{F}(A)-h_{F}\left(A^{\prime}\right)\right| \leq \mu \text {. }
$$


(ii) Suppose that $K=\mathbb{Q}$ and $A$ is semistable. Then any abelian subvariety $C$ of $A$ satisfies

$$
h(C) \leq h(A)+\frac{g}{2} \log \left(8 \pi^{2}\right) .
$$

The very recent work of Rémond [56] removes in (ii) the following two assumptions: $K=\mathbb{Q}$ and $A$ is semistable. The main ingredients for the proof of this lemma are as follows. Raynaud [54] proved Lemma 3.1 (i) for semistable abelian varieties. His proof relies on refinements of certain arguments in Faltings [21]; these refinements are due to Paršin and Zarhin. To prove (i) we reduce the problem to the semistable case established in [54]. For this reduction we use the semistability criterion of GrothendieckRaynaud [29], the criterion of Néron-Ogg-Shafarevich [63] and Dedekind's discriminant theorem. On the other hand, we deduce Lemma 3.1 (ii) from Bost's explicit lower bound for $h_{F}$ in (2.2) and a result of Ullmo-Raynaud given in [67, Proposition 3.3].

Proof of Lemma 3.1. To prove (i) we let $L=K(A[12])$ be the field of definition of the 12-torsion points of $A$. The semistable reduction criterion [29, Proposition 4.7] shows that $A_{L}$ is semistable. Let $D_{L}$ be the absolute value of the discriminant of $L$ over $\mathbb{Q}$ and let $l$ be the relative degree of $L$ over $K$. We denote by $\mathcal{T}$ the set of finite places of $L$ where $A_{L}$ has bad reduction. Let $\ell$ and $\ell^{\prime}$ be the smallest rational primes such that any place in $\mathcal{T}$ has residue characteristic different from $\ell$ and $\ell^{\prime}$. An application of [54, Théorème 4.4.9] (see (6.11) for a simplified bound) with the $L$-isogenous abelian varieties $A_{L}$ and $A_{L}^{\prime}$ implies

$$
\left|h_{F}\left(A_{L}\right)-h_{F}\left(A_{L}^{\prime}\right)\right| \leq \mu^{\prime}
$$

for $\mu^{\prime}$ an effective constant depending only on $D_{L}, l, d,|\mathcal{T}|, \ell, \ell^{\prime}$ and $g$. We now estimate these quantities effectively in terms of $g, N_{A}, d$ and $D_{K}$. The criterion of Néron-Ogg-Shafarevich [63, Theorem 1] implies that $L=K(A[12])$ is unramified over all finite places $v$ of $K$ such that $v \nmid 12$ and such that $A$ has good reduction at $v$. Thus [39, Lemma 6.2], which is based on Dedekind's discriminant theorem, gives

$$
D_{L} \leq\left(D_{K} N_{A}\right)^{l}\left(6 l^{t+2 d}\right)^{l d}
$$

for $t$ the number of finite places of $K$ where $A$ has bad reduction. It holds that $|\mathcal{T}| \leq l t$, and it is known that $l$ can be explicitly controlled in terms of $g$ (for example, one can use the embedding $\mathrm{Gal}(L / K) \hookrightarrow$ $\operatorname{Aut}\left((\mathbb{Z} / 12 \mathbb{Z})^{2 g}\right)$ induced by the action of $\operatorname{Gal}(\bar{K} / K)$ on the 12 -torsion points $A[12](\bar{K}) \cong(\mathbb{Z} / 12 \mathbb{Z})^{2 g}$ where $\bar{K}$ is an algebraic closure of $K$ ). Further, the explicit prime number theorem in [60] gives effective upper bounds for $t, \ell$ and $\ell^{\prime}$ in terms of $N_{A}$. We conclude that $\mu^{\prime}$ is bounded from above by an effective constant $\mu$ that depends only on $g, N_{A}, d$ and $D_{K}$. Then (3.2) and the stability of $h_{F}$ prove (i).

To show (ii) we assume that $K=\mathbb{Q}$ and that $A$ is semistable. Let $C$ be an abelian subvariety of $A$. Then there exists a short exact sequence

$$
0 \rightarrow C \rightarrow A \rightarrow D \rightarrow 0
$$

of abelian varieties over $\mathbb{Q}$. The semistability of $A$ provides that $C$ and $D$ are semistable as well; see, for example, [4, p. 182]. Therefore [67, Proposition 3.3] implies that $h(C) \leq h(A)-h(D)+g \log 2$ and then the lower bound for $h(D)$ given in (2.2) leads to statement (ii). This completes the proof of Lemma 3.1.

We point out that Faltings' proof of the Tate conjecture, and its refinement due to Paršin-ZarhinRaynaud [54], which is applied in Lemma 3.1 (i), both do not use Diophantine approximation or transcendence techniques. On the other hand, Masser-Wüstholz [46] gave a new proof of the Tate conjecture on using their isogeny estimates that rely on the theory of logarithmic forms from transcendence theory. Bost-David (see, for example, [6, p. 121]) showed that these isogeny estimates are fully effective, and completely explicit constants are given by Gaudron-Rémond [25]. For example, [25, Théorème 1.4] combined with (3.1) gives the following result. 
Lemma 3.2. If $A^{\prime}$ is an abelian variety over $K$ that is $K$-isogenous to $A$, then

$$
\left|h(A)-h\left(A^{\prime}\right)\right| \leq 2^{10} g^{3} \log \left((14 g)^{64 g^{2}} d \max \left(h_{F}(A), \log d, 1\right)^{2}\right) .
$$

A very recent update of (3.3) can be found in the work [27], which is discussed in Remark 5.2 (ii). On calculating the constant $\mu$ in Lemma 3.1 (i) explicitly, it turns out that Lemma 3.1 (i) improves (3.3) in some cases and vice versa in other cases; see, for example, (6.11). As already pointed out by MasserWüstholz, it is quite difficult to rigorously compare bounds coming from the two different approaches; their comparison in [46, p. 471] contains in particular an interesting discussion of certain features of the two bounds. In addition, we point out that the minimal isogeny degree estimates underlying (3.3), based on the theory of logarithmic forms, have many important Diophantine applications that are completely out of reach for (the methods underlying) Lemma 3.1.

\section{Faltings heights of Jacobians of modular curves}

In this section, we give explicit upper bounds for the stable Faltings heights of the Jacobians of certain classical modular curves in terms of their level. These upper bounds are based on a result of Javanpeykar given in [31].

We begin to state the result of Javanpeykar. Let $X$ be a smooth, projective and connected curve over $\overline{\mathbb{Q}}$ of genus $g$, where $\overline{\mathbb{Q}}$ is an algebraic closure of $\mathbb{Q}$. We denote by $\mathbb{P}^{1}$ the projective line over $\overline{\mathbb{Q}}$ and we let $\mathcal{D}$ be the set of degrees of finite morphisms $X \rightarrow \mathbb{P}^{1}$ that are unramified outside $0,1, \infty$. Belyi's theorem [2] shows that $\mathcal{D}$ is nonempty. The Belyi degree $\operatorname{deg}_{B}(X)$ of $X$ is defined by $\operatorname{deg}_{B}(X)=\min \mathcal{D}$. Let $\operatorname{Pic}^{0}(X)$ be the Jacobian of $X$, and let $h_{F}$ be the stable Faltings height defined in Section 2. We recall that $h_{F}(0)=0$ and then Javanpeykar's inequality [31, Theorem 1.1.1] gives

$$
h_{F}\left(\operatorname{Pic}^{0}(X)\right) \leq 13 \cdot 10^{6} \operatorname{deg}_{B}(X)^{5} g .
$$

We point out that $h_{F}\left(\mathrm{Pic}^{0}(X)\right)$ is well defined, because the height $h_{F}$ is stable. Next, we introduce some notation and we recall some basic results from the theory of modular curves that can be found, for example, in the books of Shimura [64] or Diamond-Shurman [16]. Let $\Gamma \subset \mathrm{SL}_{2}(\mathbb{Z})$ be a congruence subgroup. The associated modular curve has a smooth, projective and connected model $X(\Gamma)$ over $\overline{\mathbb{Q}}$. Let $g_{\Gamma}$ be the genus of $X(\Gamma)$, and let $\epsilon_{\infty}$ be the number of cusps of $X(\Gamma)$. The inclusion $\Gamma \subset \mathrm{SL}_{2}(\mathbb{Z})$ induces a natural projection $X(\Gamma) \rightarrow X(1)=X\left(\mathrm{SL}_{2}(\mathbb{Z})\right)$ whose degree $d_{\Gamma}$ satisfies

$$
g_{\Gamma} \leq 1+\frac{d_{\Gamma}}{12}-\frac{\epsilon_{\infty}}{2}, \quad d_{\Gamma}= \begin{cases}{\left[\mathrm{SL}_{2}(\mathbb{Z}): \Gamma\right]} & \text { if -id } \in \Gamma, \\ {\left[\mathrm{SL}_{2}(\mathbb{Z}): \Gamma\right] / 2} & \text { if -id } \notin \Gamma,\end{cases}
$$

where $\left[\mathrm{SL}_{2}(\mathbb{Z}): \Gamma\right]$ denotes the index of the subgroup $\Gamma \subset \mathrm{SL}_{2}(\mathbb{Z})$ and id $\in \mathrm{SL}_{2}(\mathbb{Z})$ denotes the identity. Furthermore, the projection $X(\Gamma) \rightarrow X(1)$ ramifies at most over the two elliptic points of $X(1)$ or over the cusp of $X(1)$, and it holds that $X(1) \cong \mathbb{P}^{1}$. Therefore, it follows that $\operatorname{deg}_{B}(X(\Gamma)) \leq d_{\Gamma}$ and then the displayed estimate for $h_{F}\left(\mathrm{Pic}^{0}(X)\right)$ implies

$$
h_{F}(J(\Gamma)) \leq 13 \cdot 10^{6} d_{\Gamma}^{5} g_{\Gamma}
$$

for $J(\Gamma)=\operatorname{Pic}^{0}(X(\Gamma))$ the Jacobian of $X(\Gamma)$. For any integer $N \geq 1$, we consider the classical congruence subgroups $\Gamma_{0}(N), \Gamma_{1}(N)$ and $\Gamma(N)$ of the group $\mathrm{SL}_{2}(\mathbb{Z})$ defined in [16, p. 13], and to ease notation we write $J_{0}(N)=J\left(\Gamma_{0}(N)\right), J_{1}(N)=J\left(\Gamma_{1}(N)\right)$ and $J(N)=J(\Gamma(N))$. On combining the above results, we obtain the following lemma.

Lemma 4.1. If $N \geq 1$ is an integer, then

$$
h_{F}\left(J_{0}(N)\right) \leq 7 \cdot 10^{7}(N \log N)^{6}, \quad h_{F}\left(J_{1}(N)\right) \leq 17 \cdot 10^{3} N^{12}, \quad h_{F}(J(N)) \leq 17 \cdot 10^{3} N^{18} .
$$


Proof. We recall that $h_{F}(0)=0$. Hence, to prove the claimed inequalities, we may and do assume that the Jacobians are nontrivial. Thus, the genus formulas in [16, p. 108] show that we may and do assume that $N \geq 11$ in the case $J_{1}(N)$ and that $N \geq 3$ in the cases $J_{0}(N)$ and $J(N)$. On combining (4.2) with $[38,(5.1)]$ and $\prod_{p \mid N}\left(1+\frac{1}{p}\right) \leq 1+\log N$, we obtain an upper bound for $h_{F}\left(J_{0}(N)\right)$ as stated. For $\Gamma=\Gamma_{1}(N)$ or $\Gamma=\Gamma(N)$ there exist standard formulas that express $d_{\Gamma},\left[\mathrm{SL}_{2}(\mathbb{Z}): \Gamma\right]$ and $\epsilon_{\infty}$ in terms of $N$; see, for example, [16, §3.9]. These formulas together with (4.1) and (4.2) imply upper bounds for $h_{F}\left(J_{1}(N)\right)$ and $h_{F}(J(N))$ as claimed in Lemma 4.1 .

We now include some additional details of our computations that we used here. Write $\operatorname{rad}(m)$ for the radical of $m \in \mathbb{Z}_{\geq 1}$. In the case when $\Gamma=\Gamma(N)$ and $N \geq 3$, one can use, for instance, the following formulas, which are given in [16, p.106 and p.107]:

$$
d_{\Gamma}=\frac{1}{2} N^{3} \prod\left(1-p^{-2}\right) \quad \text { and } \quad \epsilon_{\infty}=\frac{d_{\Gamma}}{N}=\frac{1}{2} \frac{N^{2}}{\operatorname{rad}(N)^{2}} \prod\left(p^{2}-1\right)
$$

with the products taken over all rational primes $p$ dividing $N$. In our situation where $N \geq 3$, these formulas imply that $d_{\Gamma} \leq \frac{1}{2} N^{3}$ and $\epsilon_{\infty} \geq 2$. In the case $\Gamma=\Gamma_{1}(N)$ and $N \geq 5$, one can use, for example, the following formulas in [16, p. 107]:

$$
d_{\Gamma}=\frac{d_{\Gamma(N)}}{N} \quad \text { and } \quad \epsilon_{\infty}=\frac{1}{2} \sum \Phi(d) \Phi(N / d)
$$

with the sum taken over all $d \in \mathbb{Z}_{\geq 1}$ dividing $N$. Here $\Phi$ is the multiplicative (Euler totient) function given by $\Phi\left(p^{k}\right)=p^{k-1}(p-1)$ for $p$ a prime and $k \in \mathbb{Z}_{\geq 1}$. In our situation where $N \geq 11$, the first formula together with the above formula for $d_{\Gamma(N)}$ implies that $d_{\Gamma} \leq \frac{1}{2} N^{2}$ and the second formula shows that $\epsilon_{\infty} \geq 2$ because $\Phi(N) \geq 4$. Indeed, $\Phi(N) \geq 4$ if $N$ has a prime factor $p \geq 5$, and if $N=2^{a} 3^{b}$ with $a, b \in \mathbb{Z}_{\geq 0}$, then we compute that $\Phi(N) \geq 4$ when $b=0$ (thus $a \geq 4$ ), when $b=1$ (thus $a \geq 2$ ) and when $b \geq 2$.

To conclude this section we discuss results in the literature that are related to Lemma 4.1. We begin with a theorem of Ullmo and we put $g=g_{\Gamma_{0}(N)}$. If $N \geq 1$ is a square-free integer, then [67, Théorème 1.2] gives the asymptotic upper bound

$$
h_{F}\left(J_{0}(N)\right) \leq \frac{g}{2} \log N+o(g \log N)
$$

Further, if $N \geq 1$ is a square-free integer, with $2 \nmid N$ and $3 \nmid N$, then Jorgenson-Kramer provide in [33, Theorem 6.2] the asymptotic formula

$$
h_{F}\left(J_{0}(N)\right)=\frac{g}{3} \log N+o(g \log N) .
$$

On combining (4.1) with the above displayed results, one can asymptotically improve the bounds for $h_{F}\left(J_{0}(N)\right)$ given in Lemma 4.1 for a special class of integers $N \geq 1$. However, our proofs of the Diophantine results in the following sections require bounds for all integers $N \geq 1$ and thus the above discussed results of Ullmo and Jorgenson-Kramer are not sufficiently general for our purpose.

\section{Abelian varieties of product $\mathrm{GL}_{2}$-type}

In the first part, we define and discuss abelian schemes of product $\mathrm{GL}_{2}$-type. Then we state inequalities relating the stable Faltings height and the conductor of abelian varieties over $\mathbb{Q}$ of product $\mathrm{GL}_{2}$-type. In the second part, we prove the height-conductor inequalities.

Let $S$ be a connected Dedekind scheme, with field of fractions $K$ a number field. Let $A$ be an abelian scheme over $S$ of relative dimension $g \geq 1$. We say that $A$ is of $\mathrm{GL}_{2}$-type if there exists a number field $F$ of degree $[F: \mathbb{Q}]=g$ together with an embedding

$$
F \hookrightarrow \operatorname{End}(A) \otimes_{\mathbb{Z}} \mathbb{Q} .
$$


The terminology $\mathrm{GL}_{2}$-type comes from the following property: If $A$ is of $\mathrm{GL}_{2}$-type and if $V_{\ell}(A)=$ $T_{\ell}(A) \otimes_{\mathbb{Z}_{\ell}} \mathbb{Q}_{\ell}$ denotes the rational $\ell$-adic Tate module associated to the generic fibre of $A$, then $V_{\ell}(A)$ is a free $F \otimes_{\mathbb{Q}} \mathbb{Q}_{\ell}$-module of rank 2 and thus the action on $V_{\ell}(A)$ of the absolute Galois group of $K$ defines a representation with values in

$$
\mathrm{GL}_{2}\left(F \otimes_{\mathbb{Q}} \mathbb{Q}_{\ell}\right) .
$$

Abelian varieties of $\mathrm{GL}_{2}$-type were studied by several authors. ${ }^{3}$ For example, we mention the fundamental contributions of Ribet [57, 59]. Further, we remark that elliptic curves and rational points on Hilbert modular varieties provide natural examples of abelian varieties of $\mathrm{GL}_{2}$-type, and there exists a vast literature on special classes (e.g., Hilbert-Blumenthal type) of abelian varieties of $\mathrm{GL}_{2}$-type; see, for instance, van der Geer [28].

More generally, we say that $A$ is of product $\mathrm{GL}_{2}$-type if $A$ is isogenous to a product $\prod A_{i}$ of nonzero abelian schemes $A_{i}$ over $S$ such that each $A_{i}$ is of $\mathrm{GL}_{2}$-type. If $A$ is isogenous to $\prod A_{i}$ and each $\operatorname{End}\left(A_{i}\right) \otimes_{\mathbb{Z}} \mathbb{Q}$ contains a number field $F_{i}$ of degree equal to the relative dimension $g_{i}$ of $A_{i}$ over $S$, then $E=\prod F_{i}$ is a commutative semisimple $\mathbb{Q}$-subalgebra of $\operatorname{End}(A) \otimes_{\mathbb{Z}} \mathbb{Q}$ with $[E: \mathbb{Q}]=g$. In the case when $K=\mathbb{Q}$, we obtain in addition the following: The abelian scheme $A$ is of product $\mathrm{GL}_{2}$-type if and only if there exists a commutative semisimple $\mathbb{Q}$-subalgebra

$$
E \hookrightarrow \operatorname{End}(A) \otimes_{\mathbb{Z}} \mathbb{Q}
$$

with $[E: \mathbb{Q}]=g$. To prove this statement, we suppose that $\operatorname{End}(A) \otimes_{\mathbb{Z}} \mathbb{Q}$ has a commutative semisimple $\mathbb{Q}$-subalgebra $E$ with $[E: \mathbb{Q}]=g$. Wedderburn's theorem gives an isomorphism of $\mathbb{Q}$-algebras $E \cong \prod F_{i}$ where each $F_{i}$ is a number field. Then, on using the idempotents of $E$, we obtain that $A$ is isogenous to a product $\prod A_{i}$ of nonzero abelian schemes $A_{i}$ over $S$ such that $F_{i}$ embeds into $\operatorname{End}\left(A_{i}\right) \otimes_{\mathbb{Z}} \mathbb{Q}$. We now use that $K=\mathbb{Q}$. By functoriality, $F_{i}$ acts on the $\mathbb{Q}$-vector space $\operatorname{Lie}\left(A_{i} \times_{S} \mathbb{Q}\right)$, which has dimension $g_{i}$. It follows that $\left[F_{i}: \mathbb{Q}\right] \leq g_{i}$. This together with the equalities $\sum g_{i}=g=\sum\left[F_{i}: \mathbb{Q}\right]$ implies that the strict inequality $\left[F_{i}: \mathbb{Q}\right]<g_{i}$ is impossible. We deduce that $\left[F_{i}: \mathbb{Q}\right]=g_{i}$, which means that each $A_{i}$ is of $\mathrm{GL}_{2}$-type. Thus, $A$ is of product $\mathrm{GL}_{2}$-type as desired.

\subsection{Height and conductor}

Let $A$ be an abelian variety over $\mathbb{Q}$ of dimension $g \geq 1$. We denote by $h_{F}(A)$ the stable Faltings height of $A$, and we denote by $N_{A}$ the conductor of $A$. See Section 2 for the definitions of $h_{F}(A)$ and $N_{A}$. We obtain the following result.

Theorem 5.1. If $A$ is of product $\mathrm{GL}_{2}$-type, then the following statements hold:

(i) There is an effective constant $k$, depending only on $g, N_{A}$, such that $h_{F}(A) \leq k$.

(ii) It holds $h_{F}(A) \leq\left(3 N_{A}\right)^{12}$.

Here (i) is a direct consequence of (ii). An advantage of first stating (i) separately is that our proof of (i) is different and this will allow us in Subsection 6.2.2 to give via (i) an interesting proof of Conjecture $(E S)$ for $A$ of product $\mathrm{GL}_{2}$-type. However, the proofs of Theorem 5.1 (i) and (ii) are in principle the same. The main difference is that in (i) we use the isogeny result in Lemma 3.1 (i) based on 'essentially algebraic' methods, and in (ii) we apply the isogeny result (3.3) based on the theory of logarithmic forms. On calculating explicitly the constant $k$ in our proof of (i), it turns out (see Remark 5.2) that the resulting bound for $h_{F}(A)$ is at least exponential in terms of $N_{A}$ and $g$. The idea underlying the proof of (i), to apply isogeny results based on essentially algebraic methods for proving height-conductor inequalities, can be useful in certain situations for improving bounds; see, for example,

\footnotetext{
${ }^{3}$ Some authors use a more restrictive definition. For example, they assume in addition simplicity or they make extra assumptions on the Lie algebra. Assuming simplicity would be too restrictive for our purpose (e.g., it would significantly weaken our results, ruling out interesting Diophantine applications). On the other hand, we do not make extra assumptions on the Lie algebra because either we do not need them or these assumptions are automatically satisfied in the situations under consideration.
} 
Proposition 6.9 and the discussion before its proof. Further, we show in Appendix A that one can refine the proof of (i) or the proof of (ii) to asymptotically improve the bound in (ii) for semistable abelian varieties with real multiplications.

\subsection{Proof of Theorem 5.1}

We first collect useful properties of abelian varieties over $\mathbb{Q}$ of $\mathrm{GL}_{2}$-type. Then we combine these properties with results obtained in Sections 2, 3 and 4.

\subsubsection{Preliminaries}

To prove Theorem 5.1 we use Serre's modularity conjecture [62, (3.2.4)?]. Building on the work of many mathematicians, Khare-Wintenberger [43] recently proved Serre's modularity conjecture. Furthermore, Ribet generalised the arguments of Serre [62, Théorème 5] and he showed in [59, Theorem 4.4] that Serre's modularity conjecure has the following consequence. Suppose that $A$ is an abelian variety over $\mathbb{Q}$ of $\mathrm{GL}_{2}$-type. If $A$ is $\mathbb{Q}$-simple, then there exists an integer $N \geq 1$ together with a surjective morphism

$$
J_{1}(N) \rightarrow A
$$

of abelian varieties over $\mathbb{Q}$. Here $J_{1}(N)$ denotes the usual modular Jacobian, defined, for example, in Section 4. We note that Serre and Ribet used the Tate conjecture [21] to prove the implication 'Serre's modularity conjecture $\Rightarrow$ (5.2)'.

We now collect additional results that will be used in the proof of Theorem 5.1. Assume that $A$ is an abelian variety over $\mathbb{Q}$ of $\mathrm{GL}_{2}$-type. Then there exists a $\mathbb{Q}$-simple abelian variety $B$ over $\mathbb{Q}$ of $\mathrm{GL}_{2}$-type, an integer $n \geq 1$ and a $\mathbb{Q}$-isogeny

$$
A \rightarrow B^{n}
$$

for $B^{n}$ the $n$-fold product of $B$. This result was established by Ribet in the course of his proof of [59, Theorem 2.1]. Further, we shall use the following important property of abelian varieties of $\mathrm{GL}_{2}$-type. Suppose that $A$ and $A^{\prime}$ are $\mathbb{Q}$-isogenous abelian varieties over $\mathbb{Q}$. Then $A$ is of $\mathrm{GL}_{2}$-type if and only if $A^{\prime}$ is of $\mathrm{GL}_{2}$-type. Indeed, this follows directly from $\operatorname{dim}(A)=\operatorname{dim}\left(A^{\prime}\right)$ and $\operatorname{End}(A) \otimes_{\mathbb{Z}} \mathbb{Q} \cong \operatorname{End}\left(A^{\prime}\right) \otimes_{\mathbb{Z}} \mathbb{Q}$.

For any abelian variety $A$ over $\mathbb{Q}$, we denote by $N_{A}$ the conductor of $A$. Let $N \geq 1$ be an integer and consider the classical congruence subgroup $\Gamma_{1}(N) \subset \mathrm{SL}_{2}(\mathbb{Z})$. For any normalised newform $f \in$ $S_{2}\left(\Gamma_{1}(N)\right)$, we let $A_{f}=J_{1}(N) / I_{f} J_{1}(N)$ be the abelian variety over $\mathbb{Q}$ associated to $f$ by Shimura's construction; see, for example, [16] for the definitions. The abelian variety $A_{f}$ is $\mathbb{Q}$-simple and the $\mathbb{Q}$-simple 'factors' of $J_{1}(N)$ are unique up to $\mathbb{Q}$-isogenies. Thus, [58, Proposition 2.3] implies that any $\mathbb{Q}$-simple quotient of $J_{1}(N)$ is $\mathbb{Q}$-isogenous to $A_{f}$ for some normalised newform $f \in S_{2}\left(\Gamma_{1}(M)\right)$ of level $M$ with $M \mid N$. Further, a result of Carayol in [10] gives for each $M \geq 1$ that any normalised newform $f \in S_{2}\left(\Gamma_{1}(M)\right)$ satisfies $N_{A_{f}}=M^{\operatorname{dim}\left(A_{f}\right)}$. Suppose now that $A$ is the $\mathbb{Q}$-simple abelian variety over $\mathbb{Q}$ of $\mathrm{GL}_{2}$-type, which appears in (5.2). Then on combining the above observations, we see that one can choose the number $N$ in (5.2) such that

$$
N_{A}=N^{\operatorname{dim}(A)} .
$$

We are now ready to prove the height-conductor inequalities.

\subsubsection{Proofs}

We continue our notation. For any abelian variety $A$ over $\mathbb{Q}$, we denote by $h_{F}(A)$ its stable Faltings height. In our proofs we shall obtain a result that is more technical than Theorem 5.1 and that we now state because it will be used again in Section 6.

Theorem 5.1*. Let $A$ be an abelian variety over $\mathbb{Q}$ of dimension $g \geq 1$. Suppose that $A$ is of product $\mathrm{GL}_{2}$-type. Then there exist positive integers $N_{i}$ and $e_{i}$, together with $\mathbb{Q}$-simple abelian subvarieties $A_{i}$ 
of $J_{1}\left(N_{i}\right)$ of dimension $g_{i}$, such that $A$ is $\mathbb{Q}$-isogenous to $\prod A_{i}^{e_{i}}$ and such that $N_{i}^{g_{i}}=N_{A_{i}}$. Furthermore, it holds that $N_{A}=\prod N_{i}^{e_{i} g_{i}}$ and

$$
h_{F}(A) \leq \sum e_{i}\left(18 \cdot 10^{3} N_{i}^{12}+(8 g)^{6} \log N_{i}\right), \quad N_{i} \geq 11 .
$$

As already mentioned, our proofs of Theorem 5.1 (i) and (ii) are essentially the same. We now describe the proof of (ii), which is divided into the following two parts. In the first part, we use (5.2) and (5.4) to show that any abelian variety $A$ as in Theorem 5.1 is $\mathbb{Q}$-isogenous to a product $\prod A_{i}^{e_{i}}$, where $e_{i} \geq 1$ is an integer and $A_{i}$ is an abelian subvariety of $J_{1}\left(N_{i}\right)$ for $N_{i} \geq 1$ an integer dividing $N_{A_{i}}$. In the second part, we then combine results from Sections 2 and 3 to deduce an upper bound for $h_{F}(A)$ in terms of $h_{F}\left(J_{1}\left(N_{i}\right)\right), \operatorname{dim}\left(J_{1}\left(N_{i}\right)\right)$ and $g=\operatorname{dim}(A)$, then in terms of $N_{i}$ and $g$ by invoking results from Section 4 and finally in terms of $N_{A}$ and $g$, because each $N_{i} \mid N_{A_{i}}$ divides $N_{A}$ by Subsection 2.2.

Proof of Theorems 5.1 and 5.1*. We take an abelian variety $A$ over $\mathbb{Q}$ of dimension $g \geq 1$ and we assume that $A$ is of product $\mathrm{GL}_{2}$-type.

1. Poincaré's reducibility theorem gives positive integers $e_{i}$ together with $\mathbb{Q}$-simple abelian varieties $A_{i}$ over $\mathbb{Q}$ such that $A$ is $\mathbb{Q}$-isogenous to the product $\prod A_{i}^{e_{i}}$. We write $g_{i}$ for the dimension of $A_{i}$. The $\mathbb{Q}$-simple 'factors' $A_{i}$ of $A$ are unique up to $\mathbb{Q}$-isogeny, and by assumption $A$ is $\mathbb{Q}$-isogenous to a product of abelian varieties over $\mathbb{Q}$ of $\mathrm{GL}_{2}$-type. Therefore, (5.3) implies that $A_{i}$ is $\mathbb{Q}$-isogenous to an abelian variety over $\mathbb{Q}$ of $\mathrm{GL}_{2}$-type and thus $A_{i}$ is of $\mathrm{GL}_{2}$-type as well. Then the results collected in (5.2) and (5.4) provide a positive integer $N_{i}$ with $N_{i}^{g_{i}}=N_{A_{i}}$ together with a surjective morphism

$$
J_{i}=J_{1}\left(N_{i}\right) \rightarrow A_{i}
$$

of abelian varieties over $\mathbb{Q}$. Let $B_{i}$ be the identity component of the kernel of $J_{i} \rightarrow A_{i}$. It is an abelian subvariety of $J_{i}$. Then Poincaré's reducibility theorem gives a complementary abelian subvariety $A_{i}^{\prime}$ of $J_{i}$ together with a $\mathbb{Q}$-isogeny $A_{i}^{\prime} \times_{\mathbb{Q}} B_{i} \rightarrow J_{i}$ induced by addition. We next verify that $A_{i}$ and $A_{i}^{\prime}$ are $\mathbb{Q}$-isogenous. The kernel of the surjective morphism $J_{i} \rightarrow A_{i}$ is a $\mathbb{Q}$-subgroup scheme of $J_{i}$ whose dimension coincides with $\operatorname{dim}\left(B_{i}\right)$. Hence, the dimension formula implies that the dimensions of $A_{i}$ and $A_{i}^{\prime}$ coincide. Let $A_{i}^{\prime} \rightarrow A_{i}$ be the morphism obtained by composing the natural inclusion $A_{i}^{\prime} \hookrightarrow A_{i}^{\prime} \times_{\mathbb{Q}} B_{i}$ with the $\mathbb{Q}$-isogeny $A_{i}^{\prime} \times_{\mathbb{Q}} B_{i} \rightarrow J_{i}$ and then with the morphism $J_{i} \rightarrow A_{i}$. We recall that the surjective morphism $A_{i}^{\prime} \times_{\mathbb{Q}} B_{i} \rightarrow J_{i}$ is induced by addition, and $B_{i}$ is the identity component of the kernel of $J_{i} \rightarrow A_{i}$. Therefore, we see that the morphism $A_{i}^{\prime} \rightarrow A_{i}$ is surjective, and thus it is a $\mathbb{Q}$-isogeny because $\operatorname{dim}\left(A_{i}^{\prime}\right)=\operatorname{dim}\left(A_{i}\right)$. Hence, after replacing $A_{i}$ by $A_{i}^{\prime}$, we may and do assume that $A_{i}$ is an abelian subvariety of $J_{i}$ and that there exists a $\mathbb{Q}$-isogeny

$$
A_{i} \times_{\mathbb{Q}} B_{i} \rightarrow J_{i}
$$

2. We now begin to estimate the heights. For any abelian variety $B$ over $\mathbb{Q}$, we denote by $v_{B}$ the maximal variation of the stable Faltings height $h_{F}$ in the $\mathbb{Q}$-isogeny class of $B$; that is, $v_{B}=$ $\sup \left|h_{F}(B)-h_{F}\left(B^{\prime}\right)\right|$ with the supremum taken over all abelian varieties $B^{\prime}$ over $\mathbb{Q}$ that are $\mathbb{Q}$-isogenous to $B$. The abelian variety $A^{\prime}=\prod A_{i}^{e_{i}}$ satisfies

$$
h_{F}\left(A^{\prime}\right)=\sum e_{i} h_{F}\left(A_{i}\right), \text { and } h_{F}(A) \leq v_{A^{\prime}}+h_{F}\left(A^{\prime}\right)
$$

because $A$ is an abelian variety over $\mathbb{Q}$ that is $\mathbb{Q}$-isogenous to $A^{\prime}$. Write $n_{i}=\operatorname{dim}\left(J_{i}\right)$ and define $J_{i}^{\prime}=A_{i} \times_{\mathbb{Q}} B_{i}$. It holds that $h_{F}\left(A_{i}\right)=h_{F}\left(J_{i}^{\prime}\right)-h_{F}\left(B_{i}\right)$, and it follows from (5.6) that $h_{F}\left(J_{i}^{\prime}\right)$ is at most $v_{J_{i}}+h_{F}\left(J_{i}\right)$. Therefore, the lower bound for $h_{F}\left(B_{i}\right)$ in (2.2) implies

$$
h_{F}\left(A_{i}\right) \leq v_{J_{i}}+h_{F}\left(J_{i}\right)+\frac{n_{i}}{2} \log \left(2 \pi^{2}\right) .
$$


Here we used that the dimension of $B_{i}$ is at most $\operatorname{dim}\left(J_{i}^{\prime}\right)=n_{i}$ and that the lower bound for $h_{F}\left(B_{i}\right)$ in (2.2) holds in addition for $B_{i}=0$ because $h_{F}(0)=0$. To control $n_{i}$ in terms of $N_{i}$, we consider the modular curve $X_{1}\left(N_{i}\right)=X\left(\Gamma_{1}\left(N_{i}\right)\right)$ over $\mathbb{Q}$ defined in Section 4. We recall that $J_{i}=J_{1}\left(N_{i}\right)$ is the Jacobian of $X_{1}\left(N_{i}\right)$ and hence the genus of $X_{1}\left(N_{i}\right)$ coincides with the dimension $n_{i}$ of $J_{i}$. Therefore, (4.1) together with [16, p. 107] implies

$$
n_{i} \leq \frac{1}{24} N_{i}^{2}
$$

To bound $N_{J_{i}}$ in terms of $N_{i}$, we use the classical result of Igusa, which says that $X_{1}\left(N_{i}\right)$ has good reduction at all primes $p \nmid N_{i}$. In particular, the Jacobian $J_{i}=\operatorname{Pic}^{0}\left(X_{1}\left(N_{i}\right)\right)$ has good reduction at all primes $p \nmid N_{i}$. It follows that all prime factors of $N_{J_{i}}$ divide $N_{i}$. Then (2.4) gives an effective bound for $N_{J_{i}}$ in terms of $n_{i}$ and $N_{i}$, which together with (5.9) shows that there exists an effective constant $c_{i}$, depending only on $N_{i}$, such that

$$
N_{J_{i}} \leq c_{i} .
$$

We recall that $A^{\prime}=\prod A_{i}^{e_{i}}$ is an abelian variety over $\mathbb{Q}$ that is $\mathbb{Q}$-isogenous to $A$. Hence, we get that $N_{A}=N_{A^{\prime}}$ and then the equality $N_{A_{i}}=N_{i}^{g_{i}}$ in statement (5.5) gives

$$
N_{A}=\prod N_{A_{i}}^{e_{i}}=\prod N_{i}^{e_{i} g_{i}} .
$$

In addition, the dimension formula gives that $g=\sum e_{i} g_{i}$. In particular, we obtain $e_{i} \leq g$.

We now prove (i). Lemma 3.1 (i) gives an effective upper bound for $v_{A^{\prime}}$ in terms of $\operatorname{dim}\left(A^{\prime}\right)=g$ and $N_{A^{\prime}}=N_{A}$ and for $v_{J_{i}}$ in terms of $n_{i}$ and $N_{J_{i}}$. On combining these upper bounds with (5.7) and (5.8), we obtain an effective estimate for $h_{F}(A)$ in terms of $g, N_{A}, n_{i}, h_{F}\left(J_{i}\right)$ and $N_{J_{i}}$; then in terms of $g, N_{A}$ and $N_{i}$ by (5.9), Lemma 4.1 and (5.10) and finally in terms of $g$ and $N_{A}$ by (5.11). This completes the proof of (i).

To show (ii) we use the inequality (3.3), which holds with $h_{F}$ in place of $h$ because the degree of an isogeny is stable. This inequality gives an upper bound for $v_{A^{\prime}}$ in terms of $\operatorname{dim}\left(A^{\prime}\right)=g$ and $h_{F}\left(A^{\prime}\right)$ and for $v_{J_{i}}$ in terms of $n_{i}$ and $h_{F}\left(J_{i}\right)$. On combining these upper bounds with (5.7) and (5.8), we obtain an estimate for $h_{F}(A)$ in terms of $g, e_{i}, n_{i}$ and $h_{F}\left(J_{i}\right)$. Then (5.9) together with the upper bound for $h_{F}\left(J_{i}\right)$ in Lemma 4.1 leads to an estimate for $h_{F}(A)$ in terms of $g, e_{i}$ and $N_{i}$. More precisely, on computing the bounds explicitly (see below for some possible intermediate steps), one obtains, for example, the bound in Theorem $5.1^{*}$ and then

$$
h_{F}(A) \leq \frac{1}{2}\left(3 N_{A}^{*}\right)^{12}+(8 g)^{6} \log N_{A}^{*}, \quad N_{A}^{*}=\prod N_{i}^{e_{i}} .
$$

To simplify the bound we used here that $N_{i}>10$. Indeed, in the cases $N_{i} \leq 10$, the modular curve $X_{1}\left(N_{i}\right)$ has genus zero and $J_{i}=\operatorname{Pic}^{0}\left(X_{1}\left(N_{i}\right)\right)=0$, which is not possible because $A_{i}$ is a nonzero abelian subvariety of $J_{i}$. Finally, it follows from (5.11) that $N_{A}^{*}$ divides $N_{A}$ and that $g \leq \frac{\log N_{A}}{\log 11}$, and then (5.12) implies (ii).

We now include some details of our computations described above in which we may and do assume that $N_{i} \geq 11$ : For example, one can use the inequalities

$$
\text { a) } \left.v_{J_{i}} \leq N_{i}^{12}, \text { b) } h_{F}\left(A_{i}\right) \leq 18 \cdot 10^{3} N_{i}^{12}, \text { c) } \sum e_{i} N_{i}^{12} \leq\left(N_{A}^{*}\right)^{12}, d\right) v_{A^{\prime}} \leq(8 g)^{6} \log \left(N_{A}^{*}\right)
$$

as intermediate steps in order to compute the bound in Theorem $5.1^{*}$, which follows from (5.7), b) and d). Here $a$ ) follows by combining (3.3), (5.9) and Lemma 4.1. Then b) follows by combining (5.8), (5.9), a) and Lemma 4.1, and c) holds because $x+y \leq x y$ for $x, y \in \mathbb{R}_{\geq 2}$. Finally, d) follows by combining (3.3), (5.7), b) and c).

Remark 5.2. (i) Without introducing new ideas, the proof of (i) gives a bound for $h_{F}(A)$ that is at least exponential in terms of $N_{A}$ and $g$. Indeed, this follows from (6.11), which shows that Lemma 3.1 (i) gives a bound for $v_{J_{i}}$ and $v_{A^{\prime}}$ that is at least exponential in terms of $n_{i}=\operatorname{dim}\left(J_{i}\right)$ and $g=\operatorname{dim}(A)$, 
respectively. The bound in (6.11) was obtained by simplifying the main result of [54]. However, any bound for $v_{J_{i}}$ and $v_{A^{\prime}}$ coming from [54] has to be at least exponential in terms of $n_{i}$ and $g$, respectively, because the crucial result [54, 4.3.7] involves a certain quantity $M \geq\left(\begin{array}{c}2 n \\ n\end{array}\right) \geq 2^{n}$ for $n \in\left\{n_{i}, g\right\}$.

(ii) The above proof shows that the (main) terms $N_{A}^{12}$ and $N_{i}^{12}$ in our bounds in Theorems 5.1 and 5.1* come from the inequality $h_{F}\left(J_{1}(N)\right) \leq 17 \cdot 10^{3} N^{e}$ in Lemma 4.1 for $e=12$. This inequality relies on [31], which can be refined in our special situation where $\operatorname{Pic}^{0}(X)=J_{1}(N)$. In case one can reduce here the exponent $e=12$ to $e<10$, one obtains further improvements of our bounds by using in addition two very recent results: A generalisation of Lemma 3.1 (ii) to arbitrary abelian varieties, which is contained in Rémond's work [56], and a refinement of (3.3) due to Gaudron-Rémond [27]. We would like to thank the referee for informing us about these recent works.

\section{Effective Shafarevich conjecture}

In the first part of this section, we discuss several aspects of the effective Shafarevich conjecture. In the second part, we give our explicit version of the effective Shafarevich conjecture for abelian varieties of product $\mathrm{GL}_{2}$-type and we deduce some applications. In the third part, we prove the results of Section 6 .

Let $S$ be a nonempty open subscheme of $\operatorname{Spec}(\mathbb{Z})$ and let $g \geq 1$ be an integer. We denote by $h_{F}(A)$ the stable Faltings height of an abelian scheme $A$ over $S$. See Section 2 for the definition. We now recall the effective Shafarevich conjecture.

Conjecture $(E S)$. There exists an effective constant c, depending only on $S$ and $g$, such that any abelian scheme A over $S$ of relative dimension $g$ satisfies $h_{F}(A) \leq c$.

As already mentioned, Conjecture $(E S)$ would have striking applications to classical Diophantine problems. For example, the following proposition gives that Conjecture $(E S)$ implies the effective Mordell conjecture for curves over number fields.

Proposition 6.1. Suppose that Conjecture (ES) holds. If $X$ is a smooth, projective and geometrically connected curve of genus at least 2 , defined over an arbitrary number field, then one can determine in principle all rational points of $X$.

Let $K$ be a number field. In what follows, by a curve over $K$ we always mean a smooth, projective and geometrically connected curve over $K$. For any curve $X$ over $K$, we denote by $h_{F}(X)$ the stable Faltings height of the Jacobian $\operatorname{Pic}^{0}(X)$ of $X$. In the first part of the proof of Proposition 6.1, we will show that Conjecture $(E S)$ implies in particular the following 'classical' effective Shafarevich conjecture $(E S)^{*}$ for curves over $K$.

Conjecture $(E S)^{*}$. Let $T$ be a finite set of places of $K$. There exists an effective constant $c$, depending only on $K, T$ and $g$, such that any curve $X$ over $K$ of genus $g$, with good reduction outside $T$, satisfies

$$
h_{F}(X) \leq c
$$

In the second part of the proof of Proposition 6.1, we apply Rémond's effective Kodaira construction in [55], which gives that $(E S)^{*}$ implies the effective Mordell conjecture.

We now discuss several aspects of Conjectures $(E S)$ and $(E S)^{*}$. First, we mention that Conjecture $(E S)$, which implies $(E S)^{*}$, is a priori considerably stronger than $(E S)^{*}$. For example, if $X$ is a curve over $K$, then Conjecture $(E S)$ would also allow controlling the finite places of $K$ where the reductions of $X$ and $\operatorname{Pic}^{0}(X)$ are different; see the discussion at the end of Section 6 for more details. Furthermore, it is shown in [40] that already special cases of Conjecture $(E S)$, such as, for example, Theorem 6.2, have direct applications to the effective study of Diophantine equations. On the other hand, one needs to prove Conjecture $(E S)^{*}$ in quite general situations to get effective Diophantine applications. For example, de Jong-Rémond [32] established Conjecture $(E S)^{*}$ for curves over $K$ that are geometrically cyclic covers of prime degree of the projective line $\mathbb{P}_{K}^{1}$ : They combined the method introduced by Paršin [52] with the theory of logarithmic forms; see also the proof of [39, Thm 3.2] for some refinements and 
$[19,24,35,37]$ for stronger results for (hyper)elliptic curves via different methods. However, the case of cyclic covers is not general enough to directly deduce applications for rational points via the known constructions of Kodaira or Paršin [51].

The proof of Proposition 6.1 shows moreover that Conjecture $(E S)$ is in fact equivalent to the following (a priori more general) conjecture: For any nonempty open subscheme $S$ of the spectrum of the ring of integers of $K$, there exists an effective constant $c$, depending only on $K, S$ and $g$, such that any abelian scheme $A$ over $S$ of relative dimension $g$ satisfies $h_{F}(A) \leq c$. To prove the equivalence one uses inter alia the Weil restriction. We refer to the proof of Proposition 6.1 for details. Further, we mention that one can, of course, formulate Conjecture $(E S)$ more classically in terms of $\mathbb{Q}$-isomorphism classes of abelian varieties over $\mathbb{Q}$ of dimension $g$, with good reduction outside a finite set of rational prime numbers. However, our formulation of Conjecture $(E S)$ in terms of abelian schemes is more convenient for the effective study of integral points on moduli schemes.

Finally, we remark that a geometric analogue of Conjecture (ES) was established by Faltings [20]; see also Deligne [15, p. 14] for some refinements.

\subsection{Abelian schemes of product $\mathrm{GL}_{2}$-type}

We continue the notation of the previous section. Let $S$ be a nonempty open subscheme of $\operatorname{Spec}(\mathbb{Z})$ and let $g \geq 1$ be an integer. We write $N_{S}=\prod p$ with the product taken over all rational prime numbers $p$ that are not in $S$. The following theorem establishes the effective Shafarevich conjecture $(E S)$ for all abelian schemes of product $\mathrm{GL}_{2}$-type.

Theorem 6.2. Let $A$ be an abelian scheme over $S$ of relative dimension $g$. If A is of product $\mathrm{GL}_{2}$-type, then

$$
h_{F}(A) \leq(3 g)^{144 g} N_{S}^{24} .
$$

We point out that the bound in Theorem 6.2 is polynomial in terms of $N_{S}$. In course of the proof of Theorem 6.2 we shall obtain the more precise inequality (6.7), which improves in particular the estimate of Theorem 6.2 and which is polynomial in terms of the relative dimension $g$ of $A$. Moreover, it is possible to refine (6.7) in special cases. For example, we obtain the following result for semistable abelian varieties of product $\mathrm{GL}_{2}$-type.

Proposition 6.3. Let $A$ be an abelian scheme over $S$ of relative dimension g. If $A$ is of product $\mathrm{GL}_{2}$-type and if the generic fibre of $A$ is semistable, then

$$
h_{F}(A) \leq g\left(3 N_{S}\right)^{12}+(6 g)^{7} \log N_{S} .
$$

Next, we deduce from Theorem 6.2 new cases of the 'classical' effective Shafarevich conjecture $(E S)^{*}$. We say that a curve $X$ over $\mathbb{Q}$ is of product $\mathrm{GL}_{2}$-type if the Jacobian $\operatorname{Pic}^{0}(X)$ of $X$ is of product $\mathrm{GL}_{2}$-type. There exist many curves over $\mathbb{Q}$ of genus $\geq 2$ that are of product $\mathrm{GL}_{2}$-type; see, for example, the articles in [13]. Let $T$ be a finite set of rational prime numbers and write $N_{T}=\prod p$ with the product taken over all $p \in T$.

Corollary 6.4. Let $X$ be a curve over $\mathbb{Q}$ of genus $g$ that is of product $\mathrm{GL}_{2}$-type. If $\mathrm{Pic}^{0}(X)$ has good reduction outside $T$, then

$$
h_{F}(X) \leq(3 g)^{144 g} N_{T}^{24} .
$$

Proof. The Néron model of $\operatorname{Pic}^{0}(X)$ over $S=\operatorname{Spec}(\mathbb{Z})-T$ is an abelian scheme, because $\operatorname{Pic}^{0}(X)$ has good reduction outside $T$. Therefore, Theorem 6.2 implies Corollary 6.4.

If $X$ is a curve over $\mathbb{Q}$ with good reduction at a rational prime $p$, then $\operatorname{Pic}^{0}(X)$ has good reduction at $p$. This shows that Corollary 6.4 establishes in particular the classical effective Shafarevich conjecture $(E S)^{*}$ for all curves over $\mathbb{Q}$ of product $\mathrm{GL}_{2}$-type. 
We now derive new isogeny estimates for abelian varieties over $\mathbb{Q}$ of product $\mathrm{GL}_{2}$-type. MasserWüstholz bounded in [46, 47] the minimal degree of isogenies of abelian varieties. On combining Theorem 6.2 with the recent version of the Masser-Wüstholz results due to Gaudron-Rémond [25], we obtain the following corollary.

Corollary 6.5. Suppose that $A$ and $B$ are isogenous abelian schemes over $S$ of relative dimension $g$. If $A$ or $B$ is of product $\mathrm{GL}_{2}$-type, then the following statements hold:

(i) There exist isogenies $A \rightarrow B$ and $B \rightarrow$ A of degree at most $(14 g)^{(12 g)^{5}} N_{S}^{(37 g)^{3}}$.

(ii) In particular, it holds that $\left|h_{F}(A)-h_{F}(B)\right| \leq(30 g)^{3} \log N_{S}+(9 g)^{6}$.

The proof of this result shows, moreover, that Corollary 6.5 (ii) holds with $h_{F}$ replaced by the relative Faltings height $h$. Further, we point out that the above isogeny estimates are independent of $A$ and $B$. As already mentioned, this is absolutely crucial for Theorem 6.6 and for certain Diophantine applications such as, for example, [40, 41]. On calculating the constant of Lemma 3.1 (i) explicitly, we see that the bound in Corollary 6.5 (ii) is better in terms of $N_{S}$ and $g$ than Lemma 3.1 (i). On the other hand, Lemma 3.1 (i) is considerably more general than Corollary 6.5 (ii).

Recall that $M_{\mathrm{GL}_{2}, g}(S)$ denotes the set of isomorphism classes of abelian schemes over $S$ of relative dimension $g$ that are of product $\mathrm{GL}_{2}$-type. Corollary 6.5 (i) is one of the main ingredients for the proof of the following quantitative finiteness result for $M_{\mathrm{GL}_{2}, g}(S)$.

Theorem 6.6. The cardinality of $M_{\mathrm{GL}_{2}, g}(S)$ is at most $(14 g)^{(9 g)^{6}} N_{S}^{(18 g)^{4}}$.

To state some consequences of (the proof of) Theorem 6.6 for $\mathbb{Q}$-isomorphism classes of abelian varieties over $\mathbb{Q}$, we recall that $T$ denotes an arbitrary finite set of rational prime numbers and we let $N_{T}=\prod_{p \in T} p$ be as above. We obtain the following corollary.

Corollary 6.7. Let $A$ be an abelian variety over $\mathbb{Q}$ of dimension $g$. We assume that $A$ has the following properties: (a) A is of product $\mathrm{GL}_{2}$-type and (b) A has good reduction outside $T$. Then the following statements hold:

(i) Up to $\mathbb{Q}$-isomorphisms, there exist at most $(14 g)^{(9 g)^{6}} N_{T}^{(18 g)^{4}}$ abelian varieties over $\mathbb{Q}$ that are $\mathbb{Q}$ isogenous to our given A.

(ii) Up to $\mathbb{Q}$-isogenies, there exist at most $(3 g)^{32 g^{2}} N_{T}^{4 g}$ abelian varieties over $\mathbb{Q}$ of dimension $g$ that have properties $(a)$ and $(b)$.

We remark that it is possible to prove a considerably more general version of Corollary 6.7 (ii) by refining Faltings' proof of [21, Satz 5] with an effective Čebotarev density theorem; see, for example, Deligne [14]. However, the resulting unconditional bound for the number of isogeny classes would be worse than the estimate in Corollary 6.7 (ii).

\subsection{Proof of the results of Section 6}

In the first part of this section, we collect some useful results for abelian schemes. In the second part, we first show Theorem 6.2, Proposition 6.3 and Corollary 6.5; then we prove Theorem 6.6 and Corollary 6.7 and finally we give the proof of Proposition 6.1.

\subsubsection{Preliminaries}

Let $S$ be a connected Dedekind scheme, with field of fractions $K$. We begin to prove some properties of morphisms of abelian schemes over $S$ that we shall use later on. Suppose that $A$ and $B$ are abelian schemes over $S$ with generic fibres $A_{K}$ and $B_{K}$, respectively. Then base change from $S$ to $K$ induces an isomorphism of abelian groups

$$
\operatorname{Hom}(A, B) \cong \operatorname{Hom}\left(A_{K}, B_{K}\right) .
$$


We now verify (6.1). Any abelian scheme over $S$ is the Néron model of its generic fibre; see, for example, [4, p. 15]. Thus, the Néron mapping property gives that any $K$-scheme morphism $\varphi_{K}: A_{K} \rightarrow B_{K}$ extends to a unique $S$-scheme morphism $\varphi: A \rightarrow B$. In addition, if $\varphi_{K}$ is a $K$-group scheme morphism, then $\varphi$ is a $S$-group scheme morphism. Therefore, we see that base change from $S$ to $K$ induces a bijection of sets $\operatorname{Hom}(A, B) \cong \operatorname{Hom}\left(A_{K}, B_{K}\right)$. Finally, base change properties of group schemes show that this bijection is in fact a homomorphism of abelian groups and hence we conclude (6.1). Furthermore, base change from $S$ to $K$ induces an isomorphism of rings

$$
\operatorname{End}(A) \cong \operatorname{End}\left(A_{K}\right) .
$$

Indeed, if $A=B$ then the isomorphism of abelian groups in (6.1) is an isomorphism of rings, because base change from $S$ to $K$ is a covariant functor from $S$-schemes to $K$-schemes.

We shall use the following property of semistable abelian varieties. Let $A$ and $B$ be abelian varieties over $K$ and let $v$ be a closed point of $S$. If there is a surjective morphism

$$
A \rightarrow B
$$

of abelian varieties over $K$ and if $A$ has semistable reduction at $v$, then $B$ has semistable reduction at $v$. We now verify this statement. Let $C$ be the reduced underlying scheme of the identity component of the kernel of $A \rightarrow B$. There exists an abelian variety $B^{\prime}$ over $K$ that is $K$-isogenous to $B$ and that fits into an exact sequence $0 \rightarrow C \rightarrow A \rightarrow B^{\prime} \rightarrow 0$ of abelian varieties over $K$. Therefore, the semistability of $A$ at $v$ together with [4, p. 182] gives that $B^{\prime}$ has semistable reduction at $v$, and then [4, p. 180] shows that $B$ is semistable at $v$ because $B$ and $B^{\prime}$ are $K$-isogenous. This proves the assertion in (6.3).

Next, we give Lemma 6.8, which will allow us later on to control the conductor of certain abelian varieties. In this lemma, we assume that $K$ is a number field with ring of integers $\mathcal{O}_{K}$ and we assume that $S$ is a nonempty open subscheme of $\operatorname{Spec}\left(\mathcal{O}_{K}\right)$. We write $d=[K: \mathbb{Q}]$ for the degree of $K$ over $\mathbb{Q}$ and we define $N_{S}=\prod N_{v}$ with the product taken over all $v \in \operatorname{Spec}\left(\mathcal{O}_{K}\right)-S$. Let $g \geq 1$ be an integer and let $\rho=\rho(S, g)$ be the number of rational primes $p$ such that $p \leq 2 g+1$ and such that there exists $v \in \operatorname{Spec}\left(\mathcal{O}_{K}\right)-S$ with $v \mid p$. If $A$ is an abelian scheme over $S$, then we denote by $N_{A}$ the conductor of $A$ defined in Subsection 2.2. The following global result in Lemma 6.8 (i) uses inter alia the local conductor estimates of Brumer-Kramer [8] stated in (2.4).

Lemma 6.8. Suppose that $A$ is an abelian scheme over $S$ of relative dimension $g$. Then the following statements hold:

(i) There exists a positive integer $v$, depending only on $K, S$ and $g$, such that $N_{A} \mid v$ and such that

$$
v \leq(2 g+1)^{6 g d \rho} N_{S}^{2 g} .
$$

(ii) If the generic fibre of $A$ is semistable, then $N_{A} \mid N_{S}^{g}$.

Proof. The generic fibre $A_{K}$ of $A$ has good reduction at all closed points of $S$, because $A$ is an abelian scheme over $S$. Therefore, $N_{A}$ takes the form

$$
N_{A}=\prod N_{v}^{f_{v}}
$$

with the product taken over all $v \in \operatorname{Spec}\left(\mathcal{O}_{K}\right)-S$, where $f_{v}=\varepsilon_{v}+\delta_{v}$ for $\varepsilon_{v}$ and $\delta_{v}$ the tame and the wild conductor of $A_{K}$ at $v$, respectively; see, for example, [61, Subsection 2.1].

We now prove (i). Let $v$ be a closed point of $\operatorname{Spec}\left(\mathcal{O}_{K}\right)$. We see that $\varepsilon_{v} \leq \operatorname{dim} V_{\ell}(A)=2 g$ for $V_{\ell}(A)$ the rational $\ell$-adic Tate module of $A_{K}$. If the residue characteristic $p$ of $v$ satisfies $p>2 g+1$, then $\delta_{v}=0$ and hence $f_{v}=\varepsilon_{v} \leq 2 g$. We denote by $b_{v}$ the right-hand side of the inequality of BrumerKramer stated in (2.4). This $b_{v}$ is an integer that depends only on $K, S, g$ and that satisfies $f_{v} \leq b_{v}$. Then we observe that $N_{A}$ divides

$$
v=N_{S}^{2 g} \prod N_{v}^{\left(b_{v}-2 g\right)}
$$


with the product taken over all $v \in \operatorname{Spec}\left(\mathcal{O}_{K}\right)-S$ of residue characteristic at most $2 g+1$. On using the definition of $b_{v}$ via (2.4), we deduce an upper bound for $b_{v}$ that then leads to an estimate for $v$ as claimed in (i). Before we include the details of the computation that we used here, we mention that one can in fact compute the precise value of $v$ for any explicitly given $K, S$ and $g$. Let $p=p_{v}$ be the residue characteristic of $v$, write $\mathfrak{e}_{v}=v(p)$ for the ramification index of $v$ and put $n=\left\lfloor\frac{2 g}{p-1}\right\rfloor$. In our computation, we used

$$
\frac{1}{\mathfrak{e}_{v}}\left(b_{v}-2 g\right)=p n+(p-1) \lambda_{p}(n) \leq 4 g+2 g\left\lfloor\frac{\log (2 g)}{\log p}\right\rfloor,
$$

which follows from $\lambda_{p}(n) \leq n\left\lfloor\frac{\log n}{\log p}\right\rfloor$ and $\frac{p}{p-1} \leq 2$. Further, any $v \in \operatorname{Spec}\left(\mathcal{O}_{K}\right)-S$ with $p_{v} \leq 2 g+1$ lies in the set $T$ of all $v \in \operatorname{Spec}\left(\mathcal{O}_{K}\right)$ with $p_{v} \mid N_{S}$ and $p_{v} \leq 2 g+1$, and it holds that $\sum_{v \in T} \mathfrak{e}_{v} \mathfrak{f}_{v}=d \rho$ and $N_{v}=p^{\mathfrak{f}_{v}}$ where $\mathfrak{f}_{v}$ denotes the residue degree of $v$. Therefore, the above displayed results show that $\log \left(v / N_{S}^{2 g}\right) \leq 2 g \sum_{v \in T} \mathfrak{e}_{v} \mathfrak{f}_{v} \log \left(p_{v}^{2} 2 g\right)$ is at most $6 g d \rho \log (2 g+1)$, because any $v \in T$ satisfies $p_{v} \leq 2 g+1$.

To show (ii) we take again a closed point $v$ of $\operatorname{Spec}\left(\mathcal{O}_{K}\right)$. Let $\mathcal{A}_{v}$ be the fibre at $v$ of the Néron model of $A_{K}$ over $\operatorname{Spec}\left(\mathcal{O}_{K}\right)$. The identity component $\mathcal{A}_{v}^{0}$ of $\mathcal{A}_{v}$ is an extension of an abelian variety $C_{v}$ by the product of a torus part $T_{v}$ with a unipotent part $U_{v}$. Let $t_{v}, u_{v}$ and $a_{v}$ be the dimensions of $T_{v}$, $U_{v}$ and $C_{v}$, respectively. It holds that $\operatorname{dim}\left(\mathcal{A}_{v}^{0}\right)=\operatorname{dim}\left(\mathcal{A}_{v}\right)=g$ and then the dimension formula gives $g=\left(t_{v}+u_{v}\right)+a_{v}$. Further, it is known that $\varepsilon_{v}=t_{v}+2 u_{v} ;$ see, for example, [29, p. 364]. Our additional assumption in (ii), that $A_{K}$ is semistable, implies that $u_{v}=0$ and $\delta_{v}=0$. Therefore, we deduce that $f_{v}=\varepsilon_{v}=t_{v}$, and this together with $t_{v} \leq t_{v}+u_{v}+a_{v}=g$ leads to $f_{v} \leq g$. Then the displayed formula for $N_{A}$ shows that $N_{A} \mid N_{S}^{g}$, which proves (ii). This completes the proof of Lemma 6.8.

We are now ready to prove the results of Section 6.

\subsubsection{Proofs}

Theorem 5.1 (i) and Lemma 6.8 directly imply Conjecture $(E S)$ for abelian schemes of product $\mathrm{GL}_{2}$ type. This proof via Theorem 5.1 (i) and Lemma 6.8 has an interesting feature discussed in the first remark after Subsection 1.2. To prove our other results, we continue the notation of the previous section. In addition, we assume that $K=\mathbb{Q}$ and that $S$ is a nonempty open subscheme of $\operatorname{Spec}(\mathbb{Z})$. Let $N_{S}$ and $g \geq 1$ be as above. Further, we assume that $A$ is an abelian scheme over $S$ of relative dimension $g$ that is of product $\mathrm{GL}_{2}$-type and we denote by $h_{F}(A)$ its stable Faltings height.

The principal ideas of the proof of Theorem 6.2 are as follows. Theorem 5.1 (ii) together with Lemma 6.8 implies directly Conjecture $(E S)$ for $A$, with an inequality of the form $h_{F}(A) \leq c(g) N_{S}^{24 g}$ for $c(g)$ a constant depending only on $g$. However, to obtain the better bound $h_{F}(A) \leq c(g) N_{S}^{24}$ and to improve the dependence on $g$ of $c(g)$, we go into the proof of Theorem 5.1 and we apply therein Lemma 6.8 with the 'simple factors' of $A$.

Proof of Theorem 6.2. 1. By assumption, $A$ is isogenous to a product of abelian schemes over $S$ that are all of $\mathrm{GL}_{2}$-type. Then (6.2) gives that the generic fibres of these abelian schemes are all of $\mathrm{GL}_{2}$-type as well. It follows that the generic fibre $A_{\mathbb{Q}}$ of $A$ is $\mathbb{Q}$-isogenous to a product of abelian varieties over $\mathbb{Q}$ of $\mathrm{GL}_{2}$-type. In other words, the abelian variety $A_{\mathbb{Q}}$ is of product $\mathrm{GL}_{2}$-type and thus satisfies all of the assumptions of Theorem 5.1.

2. We now go into the proof of Theorem 5.1. Therein we showed Theorem 5.1*, which provides positive integers $N_{i}$ and $e_{i}$, together with $\mathbb{Q}$-simple abelian varieties $A_{i}$ over $\mathbb{Q}$ of dimension $g_{i}$, such that $A_{\mathbb{Q}}$ is $\mathbb{Q}$-isogenous to $\prod A_{i}^{e_{i}}$ and such that

$$
N_{i}^{g_{i}}=N_{A_{i}}
$$

Here $N_{A_{i}}$ denotes the conductor of $A_{i}$. Furthermore, Theorem $5.1^{*}$ gives

$$
h_{F}(A) \leq \sum e_{i}\left(18 \cdot 10^{3} N_{i}^{12}+(8 g)^{6} \log N_{i}\right) .
$$


3. Next, we estimate the numbers $N_{i}$ in terms of $g$ and $S$. There exists a surjective morphism $A_{\mathbb{Q}} \rightarrow A_{i}$ of abelian varieties over $\mathbb{Q}$, and the abelian variety $A_{\mathbb{Q}}$ has good reduction at all closed points of $S$ because it extends to an abelian scheme over $S$. Therefore, [63, Corollary 2] provides that $A_{i}$ has good reduction at all closed points of $S$, and this shows that the Néron model $\mathcal{A}_{i}$ of $A_{i}$ over $S$ is an abelian scheme over $S$. Then an application of Lemma 6.8 with the abelian scheme $\mathcal{A}_{i}$ over $S$ of conductor $N_{A_{i}}$ gives that $N_{A_{i}} \leq\left(2 g_{i}+1\right)^{6 g_{i} \rho_{i}} N_{S}^{2 g_{i}}$, where $\rho_{i}=\rho\left(S, g_{i}\right)$ denotes the number of rational primes $p \notin S$ with $p \leq 2 g_{i}+1$. Further, because $A_{\mathbb{Q}}$ is $\mathbb{Q}$-isogenous to $\prod A_{i}^{e_{i}}$, we obtain

$$
g=\sum e_{i} g_{i}
$$

It follows that $g_{i} \leq g$, and this leads to $\rho_{i} \leq \rho=\rho(S, g)$. Then the above upper bound for $N_{A_{i}}$ together with (6.4) proves that $N_{i} \leq(2 g+1)^{6 \rho} N_{S}^{2}$.

4. We observe that $\rho \leq 2 g$ and (6.6) implies that $\sum e_{i} \leq g$. Thus, on combining (6.5) with the above estimate for $N_{i}$, we deduce an inequality as claimed by Theorem 6.2. To simplify the form of the final result, we may and do assume here that $g \geq 2$. Indeed, in the case $g=1$, the above arguments simplify and they give a bound that is even better than Theorem 6.2 for $g=1$; the reason is that any elliptic curve over $\mathbb{Q}$ of conductor $N$ is a quotient of the modular Jacobian $J_{0}(N)$ by [7] and Lemma 4.1 is better for $h_{F}\left(J_{0}(N)\right)$ than for $h_{F}\left(J_{1}(N)\right)$. This completes the proof of Theorem 6.2.

We recall that $\rho=\rho(S, g)$ denotes the number of rational primes $p \notin S$ with $p \leq 2 g+1$. The proof of Theorem 6.2 also gives the following more precise result: If $A$ is an abelian scheme over $S$ of relative dimension $g$ and if $A$ is of product $\mathrm{GL}_{2}$-type, then

$$
h_{F}(A) \leq g\left(18 \cdot 10^{3} v_{0}^{12}+(8 g)^{6} \log v_{0}\right), \quad v_{0}=(2 g+1)^{6 \rho} N_{S}^{2} .
$$

Let $s$ be the number of rational primes that are not in $S$. It follows that $\rho \leq s<\infty$ and then we see that (6.7) is polynomial in terms of $g$, because $s$ depends only on $S$. Furthermore, on looking, for example, at products of elliptic curves over $S$, we see that any upper bound for $h_{F}(A)$ has to be at least linear in terms of $g$. This shows that the polynomial dependence on $g$ of (6.7) is not too far from the optimal dependence on $g$. On the other hand, Lemma 6.8 implies that Frey's height conjecture [22, p. 39] would give an upper bound for $h_{F}(A)$ that is linear in terms of $\log N_{S}$, whereas (6.7) depends polynomially on $N_{S}$. We remark that an (effective) estimate for $h_{F}(A)$ that is linear in terms of $\log N_{S}$ would imply some (effective) version of the $a b c$-conjecture.

In the following proof of Proposition 6.3, we use the arguments of Theorem 6.2 and we replace therein Lemma 6.8 (i) by Lemma 6.8 (ii).

Proof of Proposition 6.3. We freely use the notations and definitions of the proof of Theorem 6.2. In addition, we assume that $A_{\mathbb{Q}}$ is semistable. Therefore, (6.3) implies that $A_{i}$ is semistable, because there exists a surjective morphism $A_{\mathbb{Q}} \rightarrow A_{i}$ of abelian varieties over $\mathbb{Q}$. We showed that $A_{i}$ extends to an abelian scheme $\mathcal{A}_{i}$ over $S$. Thus, an application of Lemma 6.8 (ii) with the abelian scheme $\mathcal{A}_{i}$ over $S$ of conductor $N_{A_{i}}$ and relative dimension $g_{i}$ gives that $N_{A_{i}} \mid N_{S}^{g_{i}}$. Hence, the equality $N_{i}^{g_{i}}=N_{A_{i}}$ in (6.4) implies that $N_{i} \leq N_{S}$ and then (6.6) together with the upper bound for $h_{F}(A)$ in (6.5) leads to an inequality as claimed. This completes the proof of Proposition 6.3.

To prove Corollary 6.5 we combine Theorem 6.2 with the recent version of the Masser-Wüstholz results [46, 47] due to Gaudron-Rémond [25].

Proof of Corollary 6.5. We suppose that $A$ and $B$ are isogenous abelian schemes over $S$ of relative dimension $g$. Let $A_{\mathbb{Q}}$ and $B_{\mathbb{Q}}$ be the generic fibres of $A$ and $B$, respectively. By assumption, $A$ or $B$ is of product $\mathrm{GL}_{2}$-type. Thus, both are of product $\mathrm{GL}_{2}$-type.

To show (i) we observe that the constant $\kappa\left(A_{\mathbb{Q}}\right)$ in [25] depends only on $g$ and $h_{F}(A)$. Let $\kappa$ be the constant that one obtains by replacing the number $h_{F}(A)$ with $(3 g)^{144 g} N_{S}^{24}$ in the definition of $\kappa\left(A_{\mathbb{Q}}\right)$; notice that $\kappa$ depends only on $N_{S}$ and $g$. An application of Theorem 6.2 with $A$ shows that $\kappa\left(A_{\mathbb{Q}}\right) \leq \kappa$. 
The abelian varieties $A_{\mathbb{Q}}$ and $B_{\mathbb{Q}}$ are $\mathbb{Q}$-isogenous. Therefore, [25, Théorème 1.4] gives $\mathbb{Q}$-isogenies $\varphi_{\mathbb{Q}}: A_{\mathbb{Q}} \rightarrow B_{\mathbb{Q}}$ and $\psi_{\mathbb{Q}}: B_{\mathbb{Q}} \rightarrow A_{\mathbb{Q}}$ of degree at most $\kappa\left(A_{\mathbb{Q}}\right) \leq \kappa$. As in the proof of (6.1) we see that $\varphi_{\mathbb{Q}}$ and $\psi_{\mathbb{Q}}$ extend to $S$-group scheme morphisms $\varphi: A \rightarrow B$ and $\psi: B \rightarrow A$, respectively. Furthermore, it follows from [4] that $\varphi$ and $\psi$ are isogenies because $A$ and $B$ are abelian (and thus semi-abelian) schemes over $S$. Hence, we conclude (i).

It remains to prove (ii). We showed in (i) that there is a $\mathbb{Q}$-isogeny $\varphi_{\mathbb{Q}}: A_{\mathbb{Q}} \rightarrow B_{\mathbb{Q}}$ with $\operatorname{deg}\left(\varphi_{\mathbb{Q}}\right) \leq \kappa$, and (3.1) gives that $\left|h\left(A_{\mathbb{Q}}\right)-h\left(B_{\mathbb{Q}}\right)\right| \leq \frac{1}{2} \log \operatorname{deg}\left(\varphi_{\mathbb{Q}}\right)$ for $h$ the relative Faltings height. Hence, we deduce a version of (ii) involving $h$. To prove the version involving $h_{F}$ we use [29]. It provides a number field $L$ such that $A_{L}$ and $B_{L}$ are semistable, and thus $h_{F}(A)=h\left(A_{L}\right)$ and $h_{F}(B)=h\left(B_{L}\right)$. If $\varphi_{L}: A_{L} \rightarrow B_{L}$ is the base change of $\varphi_{\mathbb{Q}}$, then (3.1) gives that $\left|h\left(A_{L}\right)-h\left(B_{L}\right)\right| \leq \frac{1}{2} \log \operatorname{deg}\left(\varphi_{L}\right)$. Therefore, $\operatorname{deg}\left(\varphi_{L}\right)=\operatorname{deg}\left(\varphi_{\mathbb{Q}}\right) \leq \kappa$ leads to (ii). This completes the proof of Corollary 6.5.

We refer to the introduction for an outline of the strategy used in the following proof.

Proof of Theorem 6.6. We recall that $M_{\mathrm{GL}_{2}, g}(S)$ denotes the set of isomorphism classes of abelian schemes over $S$ of relative dimension $g$ that are of product $\mathrm{GL}_{2}$-type. To bound $\left|M_{\mathrm{GL}_{2}, g}(S)\right|$ we may and do assume that the set $M_{\mathrm{GL}_{2}, g}(S)$ is not empty.

1 . We denote by $M_{\mathrm{GL}_{2}, g}(S)_{\mathbb{Q}}$ the set of $\mathbb{Q}$-isomorphism classes of abelian varieties over $\mathbb{Q}$ of dimension $g$ that extend to an abelian scheme over $S$ and that are of product $\mathrm{GL}_{2}$-type. Base change from $S$ to $\mathbb{Q}$ induces a canonical bijection

$$
M_{\mathrm{GL}_{2}, g}(S) \cong M_{\mathrm{GL}_{2}, g}(S)_{\mathbb{Q}}
$$

To verify this statement we observe that $M_{\mathrm{GL}_{2}, g}(S)$ coincides with the set of $S$-scheme isomorphism classes generated by abelian schemes over $S$ of relative dimension $g$ that are of product $\mathrm{GL}_{2}$-type. Further, it follows from (6.2) that the generic fibre $A_{\mathbb{Q}}$ of any $[A] \in M_{\mathrm{GL}_{2}, g}(S)$ is of product $\mathrm{GL}_{2}$-type. Thus, base change from $S$ to $\mathbb{Q}$ induces a map $M_{\mathrm{GL}_{2}, g}(S) \rightarrow M_{\mathrm{GL}_{2}, g}(S)_{\mathbb{Q}}$, which is surjective by (6.2) and [4, p. 180]. The abelian scheme $A$ is the Néron model of $A_{\mathbb{Q}}$ over $S$ and then the Néron mapping property shows that $M_{\mathrm{GL}_{2}, g}(S) \rightarrow M_{\mathrm{GL}_{2}, g}(S)_{\mathbb{Q}}$ is injective. We conclude that $M_{\mathrm{GL}_{2}, g}(S) \cong M_{\mathrm{GL}_{2}, g}(S)_{\mathbb{Q}}$.

2. Next, we estimate the number of distinct $\mathbb{Q}$-isogeny classes of abelian varieties over $\mathbb{Q}$ generated by $M_{\mathrm{GL}_{2}, g}(S)_{\mathbb{Q}}$. Let $[A] \in M_{\mathrm{GL}_{2}, g}(S)_{\mathbb{Q}}$. Theorem 5.1* gives positive integers $N_{i}$ and $e_{i}$, together with $\mathbb{Q}$-simple abelian varieties $A_{i}$ over $\mathbb{Q}$ of dimension $g_{i}$ and of conductor $N_{A_{i}}=N_{i}^{g_{i}}$, such that $A$ is $\mathbb{Q}$ isogenous to $\prod A_{i}^{e_{i}}$ and such that $A_{i}$ is an abelian subvariety of $J_{1}\left(N_{i}\right)$. Here $J_{1}(N)$ denotes the usual modular Jacobian of level $N \in \mathbb{Z}_{\geq 1}$ defined in Section 4 . The abelian variety $A$ extends to an abelian scheme over $S$, because $[A] \in M_{\mathrm{GL}_{2}, g}(S)_{\mathbb{Q}}$. Thus, each $A_{i}$ extends to an abelian scheme over $S$ and then the arguments of the proof of Lemma 6.8 together with $g_{i} \leq g$ lead to $N_{A_{i}} \mid v^{g_{i}}$ for

$$
v=N_{S}^{2} \prod p^{c_{p}}, \quad c_{p}=4+2\lfloor\log (2 g) / \log p\rfloor .
$$

Here the product is taken over all rational primes $p \notin S$ with $p \leq 2 g+1$, and for any real number $x$ we recall that $\lfloor x\rfloor$ denotes the largest integer at most $x$. We warn the reader that the displayed number $v$ is related to the number appearing in Lemma 6.8 (i), but these numbers are not necessarily the same. It follows that $N_{i} \mid v$ because $N_{i}^{g_{i}}=N_{A_{i}}$, and this implies that $J_{1}\left(N_{i}\right)$ is a $\mathbb{Q}$-quotient of $J_{1}(v)$. On using that $A_{i}$ is an abelian subvariety of $J_{1}\left(N_{i}\right)$, we then see that there exists a surjective morphism of abelian varieties over $\mathbb{Q}$

$$
J_{1}(v) \rightarrow A_{i}
$$

Hence, Poincare's reducibility theorem shows that each $A_{i}$ is $\mathbb{Q}$-isogenous to a $\mathbb{Q}$-simple 'factor' of $J_{1}(v)$. Furthermore, the dimension of $J_{1}(v)$ coincides with the genus $g_{v}$ of the modular curve $X_{1}(v)=X\left(\Gamma_{1}(v)\right)$ defined in Section 4 , and the abelian variety $J_{1}(v)$ (respectively $A$ ) has at most $g_{v}$ (respectively $g$ ) $\mathbb{Q}$ simple factors up to $\mathbb{Q}$-isogenies. Therefore, there exists a set of at most $g \cdot g_{v}^{g}$ distinct abelian varieties over $\mathbb{Q}$ such that any $[A] \in M_{\mathrm{GL}_{2}, g}(S)_{\mathbb{Q}}$ is $\mathbb{Q}$-isogenous to some abelian variety in this set. In other 
words, the abelian varieties in $M_{\mathrm{GL}_{2}, g}(S)_{\mathbb{Q}}$ generate at most $g \cdot g_{v}^{g}$ distinct $\mathbb{Q}$-isogeny classes of abelian varieties over $\mathbb{Q}$.

As pointed out by the referee, one can remove here the factor $g$ by using in addition the following combinatorial counting result: For any given integers $l, m, c_{1}, \ldots, c_{m}$ in $\mathbb{Z}_{\geq 1}$ and $n=l+m-1$, there exist at most $\left(\begin{array}{l}n \\ l\end{array}\right) \leq m^{l}$ distinct $e \in \mathbb{Z}_{\geq 0}^{m}$ with $\sum e_{i} c_{i}=l$. This is then applied with $l=g, m$ the number of $\mathbb{Q}$-simple factors $C_{i}$ of $J_{1}(v)$ up to $\mathbb{Q}$-isogenies and $c_{i}=\operatorname{dim}\left(C_{i}\right)$. The combinatorial counting result can be verified as follows. We denote by $\Sigma$ the set of all $e \in \mathbb{Z}_{\geq 0}^{m}$ with $\sum e_{i} c_{i}=l$. For any $e \in \Sigma$, we define $\iota(e) \in\{0,1\}^{n}$ as follows: The first $e_{1} c_{1}$-entries of $\iota(e)$ are 0 followed by an entry that is 1 ; then the next $e_{2} c_{2}$-entries are again 0 followed by an entry that is 1 and so on until all $n$ entries of $\iota(e)$ are defined. Then $e \mapsto \iota(e)$ defines an injective map from $\Sigma$ into the set $W$ of all $w \in\{0,1\}^{n}$ such that $w$ has precisely $l$ distinct entries that are 0 . Therefore, we conclude that $|\Sigma| \leq|W|=\left(\begin{array}{l}n \\ l\end{array}\right)$ as desired.

3. To bound the size of each $\mathbb{Q}$-isogeny class we take an arbitrary $[A] \in M_{\mathrm{GL}_{2}, g}(S)_{\mathbb{Q}}$. We denote by $\mathcal{C}$ the set of $\mathbb{Q}$-isomorphism classes of abelian varieties over $\mathbb{Q}$ that are $\mathbb{Q}$-isogenous to $A$. Let $\kappa$ be the constant that appears in the proof of Corollary 6.5. If $[B] \in \mathcal{C}$, then the proof of Corollary 6.5 provides a $\mathbb{Q}$-isogeny $\varphi: A \rightarrow B$ of degree at most $\kappa$. Furthermore, the quotient of $A$ by the kernel of $\varphi$ is an abelian variety over $\mathbb{Q}$ that is $\mathbb{Q}$-isomorphic to $B$. On combining the above observations, we see that $|\mathcal{C}|$ is bounded from above by the number of subgroups of $A^{t}$ of order at most $\kappa$, where $A^{t}$ is the group of geometric torsion points of $A$. It holds that $A^{t} \cong(\mathbb{Q} / \mathbb{Z})^{2 g}$, and $\left[46\right.$, Lemma 6.1] gives that $(\mathbb{Q} / \mathbb{Z})^{2 g}$ has at most $\kappa^{2 g}$ subgroups of order at most $\kappa$. This implies that $|\mathrm{C}| \leq \kappa^{2 g}$.

4. The results obtained in 1-3 imply that $\left|M_{\mathrm{GL}_{2}, g}(S)\right| \leq g\left(g_{v} \kappa^{2}\right)^{g}$, and (4.1) together with [16, p. 107] proves that $g_{v} \leq \frac{1}{24} v^{2}$. Therefore, the definitions of $\kappa$ and $v$ lead to an upper bound for $\left|M_{\mathrm{GL}_{2}, g}(S)\right|$ as claimed in Theorem 6.6.

The arguments used in the proof of Theorem 6.6 also give Corollary 6.7.

Proof of Corollary 6.7. We observe that part 3 of the proof of Theorem 6.6 implies (i), and we notice that (ii) follows from part 2 of the proof of Theorem 6.6.

It remains to prove Proposition 6.1. In the first part of the proof we show that Conjecture $(E S)$ implies Conjecture $(E S)^{*}$, and in the second part we use the effective version of the Kodaira construction due to Rémond [55].

Proof of Proposition 6.1. We recall some notation. Let $K$ be a number field of degree $d=[K: \mathbb{Q}]$, with ring of integers $\mathcal{O}_{K}$. We denote by $D_{K}$ the absolute value of the discriminant of $K$ over $\mathbb{Q}$. Let $h_{F}$ be the stable Faltings height and let $T$ be a finite set of places of $K$. We write $N_{T}=\prod N_{v}$ with the product taken over all finite places $v \in T$. Let $X$ be a smooth, projective and geometrically connected curve over $K$ of genus $g \geq 1$.

1. To prove that Conjecture $(E S)$ implies $(E S)^{*}$ we assume that Conjecture $(E S)$ holds. In addition, we suppose that the Jacobian $J_{K}=\operatorname{Pic}^{0}(X)$ of $X$ has good reduction outside $T$. The Weil restriction $A_{\mathbb{Q}}=\operatorname{Res}_{K / \mathbb{Q}}\left(J_{K}\right)$ of $J_{K}$ is an abelian variety over $\mathbb{Q}$ of dimension $n=d g$, which is geometrically isomorphic to $\prod J_{K}^{\sigma}$. Here the product is taken over all embeddings $\sigma$ from $K$ into an algebraic closure of $K$, and $J_{K}^{\sigma}$ is the base change of $J_{K}$ with respect to $\sigma$. The Galois invariance $h_{F}\left(J_{K}\right)=h_{F}\left(J_{K}^{\sigma}\right)$ implies that $h_{F}\left(A_{\mathbb{Q}}\right)=d h_{F}\left(J_{K}\right)$. Let $S$ be the open subscheme of $\operatorname{Spec}(\mathbb{Z})$ formed by the generic point together with the closed points where $A_{\mathbb{Q}}$ has good reduction. The Néron model $A$ of $A_{\mathbb{Q}}$ over $S$ is an abelian scheme. Therefore, an application of Conjecture $(E S)$ with $A, S$ and $n$ gives an effective constant $c$, depending only on $S$ and $n$, such that

$$
d h_{F}\left(J_{K}\right)=h_{F}\left(A_{\mathbb{Q}}\right) \leq c .
$$

We write $\mathcal{D}=\left\{D_{K}, d, g, N_{T}\right\}$ and we now construct an effective constant $c^{\prime}$, depending only on $\mathcal{D}$, such that $c \leq c^{\prime}$. The finite places in $T$ form a closed subset of $\operatorname{Spec}\left(\mathcal{O}_{K}\right)$, whose complement $S^{\prime}$ has the structure of an open subscheme of $\operatorname{Spec}\left(\mathcal{O}_{K}\right)$. The Néron model $J$ of $J_{K}$ over $S^{\prime}$ is an abelian scheme, because $J_{K}$ has good reduction outside $T$. We denote by $N_{J}$ and $N_{A}$ the conductors of $J_{K}$ and 
$A_{\mathbb{Q}}$, respectively. A result of Milne [49, Proposition 1] gives that $N_{A}=N_{J} D_{K}^{2 g}$, and an application of Lemma 6.8 (i) with the abelian scheme $J$ over $S^{\prime}$ of relative dimension $g$ implies that $N_{J} \leq \Omega D_{K}^{-2 g}$ for $\Omega=(3 g)^{12 g^{2} d}\left(N_{T} D_{K}\right)^{2 g}$. We deduce that $N_{A} \leq \Omega$, and this leads to

$$
N_{S} \leq \Omega
$$

because $N_{S}$ divides $N_{A}$ by the construction of $S$. Here for any open subscheme $U$ of $\operatorname{Spec}(\mathbb{Z})$ we write $N_{U}=\prod p$ with the product taken over all rational primes $p \notin U$. It follows that $S \in \mathcal{U}$ for $\mathcal{U}$ the set of open subschemes $U$ of $\operatorname{Spec}(\mathbb{Z})$ with $N_{U} \leq \Omega$. An application of Conjecture $(E S)$ with $U \in \mathcal{U}$ and $n$ gives an effective constant $c_{U} \geq 1$, depending only on $U$ and $n$. We define $c^{\prime}=\max c_{U}$ with the maximum taken over all $U \in \mathcal{U}$. If $\mathcal{D}$ is given, then the set $\mathcal{U}$ can be determined effectively. Thus, we see that $c^{\prime}$ is an effective constant, depending only on $\mathcal{D}$. On using that $S \in \mathcal{U}$, we obtain that $c \leq c^{\prime}$ and then (6.8) gives

$$
h_{F}\left(J_{K}\right) \leq c^{\prime}
$$

In other words, we proved that Conjecture $(E S)$ would give an effective constant $c^{\prime}$, depending only on $\mathcal{D}$, with the following property: If $J_{K}$ has good reduction outside $T$, then $h_{F}\left(J_{K}\right) \leq c^{\prime}$. Further, if $X$ has good reduction at a finite place $v$ of $K$, then $J_{K}$ has good reduction at $v$. Therefore, we conclude that Conjecture $(E S)$ implies $(E S)^{*}$.

2. It follows from part 1 that Conjecture $(E S)$ implies $(E S)^{*}$. Furthermore, [55] gives that Conjecture $(E S)^{*}$ implies that the set of rational points of $X$ can be determined effectively if $g \geq 2$. This completes the proof of Proposition 6.1.

We remark that the above proof of Proposition 6.1 assumes the validity of Conjecture $(E S)$ in quite general situations. In particular, it is a priori not possible to use the above arguments in order to deduce special cases of the effective Mordell conjecture from special cases of Conjecture (ES) such as, for example, Theorem 6.2. To 'transfer' special cases between these conjectures, an effective version of Paršin's construction [51] would be more useful than Kodaira's construction, which is used in the proof of Proposition 6.1.

We mention that the implication $(E S)^{*} \Rightarrow(E S)$ remains an interesting open problem, which is nontrivial because $(E S)$ is a priori considerably stronger than $(E S)^{*}$. To discuss parts of the additional information contained in Conjecture $(E S)$, we consider an arbitrary hyperelliptic curve $X$ of genus $g \geq 2$ over a number field $K$. Let $T$ be the set of finite places of $K$ where $\operatorname{Pic}^{0}(X)$ has bad reduction. Suppose that $v$ is a finite place of $K$ where $X$ has bad reduction but $\operatorname{Pic}^{0}(X)$ has good reduction; the minimal regular model of $X$ over $\operatorname{Spec}\left(\mathcal{O}_{v}\right)$ is then automatically semistable for $\mathcal{O}_{v}$ the local ring at $v$. Then on combining the arguments of [39, Proposition 5.1 (i)] with part 1 of the proof of Proposition 6.1, we see that already very special cases of Conjecture $(E S)$ would give an effective estimate for $N_{v}$ in terms of $K, g$ and $T$. We note that Levin [44] proved that such an effective estimate for $N_{v}$ would solve the following classical problem: Give an effective version of Siegel's theorem for arbitrary hyperelliptic curves of genus $g \geq 2$ defined over a number field $K$. In fact, the latter problem is already open for $g=2$ and $K=\mathbb{Q}$.

\section{Appendix A: A height-conductor inequality for semistable abelian varieties over $\mathbb{Q}$ with real multiplications}

This appendix generalises the asymptotic bound [36, Prop 8.2] for semistable elliptic curves to semistable abelian varieties with real multiplications. In particular, for such abelian varieties we show that one can refine the proof of Theorem 5.1 (i) or (ii) in order to asymptotically improve the bound in Theorem 5.1 (ii).

Continue the notation of Sections 4 and 5. Let $A$ be an abelian variety over $\mathbb{Q}$ of dimension $g \geq 1$. We say that $A$ is with real multiplications if $\operatorname{End}(A) \otimes_{\mathbb{Z}} \mathbb{Q}$ has a $\mathbb{Q}$-subalgebra of dimension $g$ that is the product of totally real number fields. This generalises the definition of Ribet [57] and Serre [62], who 
studied the case when $A$ is $\mathbb{Q}$-simple. If $A$ is with real multiplications, then (5.1) gives that $A$ is of product $\mathrm{GL}_{2}$-type. Write $\operatorname{rad}(m)$ for the radical of $m \in \mathbb{Z}_{\geq 1}$ and define $f(x)=x \log (x) \log \log x$ for $x \in \mathbb{R}_{>1}$.

Proposition 6.9. Suppose that $A$ is of product $\mathrm{GL}_{2}$-type. Assume, moreover, that $A$ is with real multiplications and that $A$ is semistable. Then the following statements hold:

(i) Let $\gamma=0.5772 \ldots$ be Euler's constant and put $n=\operatorname{rad}\left(N_{A}\right)$. If $n \rightarrow \infty$, then

$$
h_{F}(A) \leq \frac{e^{\gamma}}{u \pi^{2}} g f(n)+o_{g}(f(n)) .
$$

Here $u=6$ if $\operatorname{gcd}(n, 6)=1$ and $u=4$ otherwise. Further, $o_{g}(f(n))=c(g) o(f(n))$ where $c(g)$ is a constant depending only $g$.

(ii) It holds that $h_{F}(A) \ll_{\epsilon} N_{A}^{1+\epsilon}$.

The bound in (ii) and the estimate (6.9), which we obtained in a more precise form in (6.10), both asymptotically improve the bound in Theorem 5.1 (ii). On the other hand, Theorem 5.1 (ii) makes no additional semistable or real multiplication assumptions, and this is crucial for many Diophantine applications (e.g., [40]). In view of this, it would be interesting to remove in Proposition 6.9 the semistable and real multiplication assumptions. Unfortunately, in the proof we use both assumptions in order to work with $X_{0}(N)=X\left(\Gamma_{0}(N)\right)$ with $N$ square-free: This allows us to apply results of Ullmo [67] and Jorgenson-Kramer [33], whose proofs crucially exploit that the modular curve is $X_{0}(N)$ with $N$ square-free. To remove any of these two assumptions, one has to overcome various technical (and also conceptual) difficulties; see, for example, the discussions in Coleman-Edixhoven [12, Sect 5], the proof of Edixhoven-de Jong [17] dealing with the case $A=J_{1}(p q)$ for $p, q$ distinct rational primes or Mayer's result [48] on $h_{F}\left(J_{1}(N)\right)$ for some square-free $N$.

To prove Proposition 6.9 (i), we refine the proof of Theorem 5.1 (i) for semistable abelian varieties with real multiplications by applying Lemma 3.1 (ii) and the bounds for $h_{F}\left(J_{0}(N)\right)$ in (4.3) and (4.4) obtained by Ullmo [67] and Jorgenson-Kramer [33], respectively. Here one can replace Lemma 3.1 (i) by (3.3). This has the advantage that one obtains a constant $c(g)$ in $o_{g}(f(n))$ that is polynomial in terms of $g$ and thus allows deducing Proposition 6.9 (ii). We shall see below that Lemma 3.1 (ii) based on 'essentially algegraic' methods is crucial for obtaining the bounds in Proposition 6.9 (i) and (ii), and in view of (6.11) the result (3.3) based on the theory of logarithmic forms is crucial for obtaining the bound in Proposition 6.9 (ii).

Proof of Proposition 6.9. As in the statement of the proposition, we assume that $A$ is with real multiplications and that $A$ is semistable. In [36, Prop 8.2] we proved Proposition 6.9 in the case $g=1$. However, we now show that the proof works for any $g \geq 2$ when combined with our arguments of Theorem 5.1. The details are as follows.

We first prove (i). Our assumption that $A$ is with real multiplications assures that $\operatorname{End}(A) \otimes_{\mathbb{Z}} \mathbb{Q}$ contains a $\mathbb{Q}$-subalgebra $E=\prod F_{i}$ with $[E: \mathbb{Q}]=g$, where each $F_{i}$ is a totally real number field. On using the idempotents of $E$, we obtain abelian varieties $B_{i}$ over $\mathbb{Q}$ such that $F_{i}$ is contained in $\operatorname{End}\left(B_{i}\right) \otimes_{\mathbb{Z}} \mathbb{Q}$ and such that $A$ is $\mathbb{Q}$-isogenous to the product $\prod B_{i}$. Then, as in the proof of (5.1), we see that a Lie algebra argument together with $[E: \mathbb{Q}]=g$ implies that $\left[F_{i}: \mathbb{Q}\right]=\operatorname{dim}\left(B_{i}\right)$ and hence each $B_{i}$ is of $\mathrm{GL}_{2}$-type. Then (5.3) gives an integer $e_{i}$ together with a $\mathbb{Q}$-simple abelian variety $A_{i}$ over $\mathbb{Q}$ of $\mathrm{GL}_{2}$-type such that $B_{i}$ is $\mathbb{Q}$-isogenous to $A_{i}^{e_{i}}$. Moreover, Ribet's arguments in the proof of [59, Thm 2.1] show that $\operatorname{End}\left(A_{i}\right) \otimes_{\mathbb{Z}} \mathbb{Q}$ is a number field of degree $\operatorname{dim}\left(A_{i}\right)$ that is a subfield of $F_{i}$ and hence is totally real. We conclude that each abelian variety $A_{i}$ is with real multiplications in the sense of Serre [62, Subsect 4.7]. Hence, Serre's modularity conjecture [43] combined with [62, Thm 5] provides a positive integer $N_{i}$ with $N_{i}^{g_{i}}=N_{A_{i}}$ together with a surjective morphism $J_{0}\left(N_{i}\right) \rightarrow A_{i}$ of abelian varieties over $\mathbb{Q}$, where $g_{i}=\operatorname{dim}\left(A_{i}\right)$. On repeating word by word the (general) arguments given below (5.5), we deduce the following: After possibly replacing $A_{i}$ by a $\mathbb{Q}$-isogenous abelian variety over $\mathbb{Q}$, we may and do assume that $A_{i}$ is an abelian subvariety of $J_{i}=J_{0}\left(N_{i}\right)$. To estimate the heights, we recall that $v_{A^{\prime}}$ 
denotes the maximal variation of the stable Faltings height $h_{F}$ in the isogeny class of the abelian variety $A^{\prime}=\prod A_{i}^{e_{i}}$ over $\mathbb{Q}$. It holds that

$$
h_{F}\left(A^{\prime}\right)=\sum e_{i} h_{F}\left(A_{i}\right) \text { and } h_{F}(A) \leq v_{A^{\prime}}+h_{F}\left(A^{\prime}\right)
$$

because $A$ is an abelian variety over $\mathbb{Q}$ that is $\mathbb{Q}$-isogenous to $A^{\prime}$. Our assumption that $A$ is semistable implies that the $\mathbb{Q}$-isogenous $A^{\prime}$ is semistable as well. In particular, the factors $A_{i}$ of $A^{\prime}$ are all semistable and thus the level $N_{i}$ is square-free. Indeed, the square-free claim follows, for example, from Lemma 6.8 (ii), which gives that the conductor $N_{A_{i}}=N_{i}^{g_{i}}$ of the semistable abelian variety $A_{i}$ divides $\left(\prod p\right)^{g_{i}}$ with the product taken over all bad reduction primes of $A_{i}$. Now, because $N_{i}$ is square-free, we obtain that $J_{i}$ is semistable and then an application of Lemma 3.1 (ii) with the abelian subvariety $A_{i}$ of $J_{i}$ gives

$$
h_{F}\left(A_{i}\right) \leq h_{F}\left(J_{i}\right)+\frac{n_{i}}{2} \log \left(8 \pi^{2}\right)
$$

where $n_{i}=\operatorname{dim}\left(J_{i}\right)$. To control the variation $v_{A^{\prime}}$, we use again our assumption that $A$ is semistable and we exploit that the $\mathbb{Q}$-isogenous $A^{\prime}$ has good reduction outside the set $T$ of rational primes dividing $N_{A}=N_{A^{\prime}}$. Then, on applying the explicit bound (3.2) for $\left|h_{F}(A)-h_{F}\left(A^{\prime}\right)\right|$ of Raynaud [54, Thm 4.4.9] with $A^{\prime}, T$ and on using the prime number theorem, we deduce that

$$
v_{A^{\prime}} \leq \kappa n,
$$

where $\kappa$ is a constant depending only on $g$. The genus of the modular curve $X_{0}\left(N_{i}\right)$ coincides with the dimension $n_{i}$ of its Jacobian $J_{i}=J_{0}\left(N_{i}\right)$. Therefore, we obtain that $n_{i} \leq \frac{e^{\gamma}}{2 \pi^{2}} N_{i} \log \log \left(N_{i}\right)+o\left(N_{i}\right)$; see, for example, the proof of [36, Prop 8.2]. Then, on applying Ullmo's asymptotic bound (4.3) or the asymptotic formula (4.4) of Jorgenson-Kramer when 6 is coprime to $n$, we see that the above displayed results lead to

$$
h_{F}(A) \leq \kappa n+\frac{e^{\gamma}}{u \pi^{2}} \sum e_{i} f\left(N_{i}\right)+o\left(f\left(N_{i}\right)\right) \leq \frac{e^{\gamma}}{u \pi^{2}} \sum e_{i} f\left(N_{A_{i}}^{1 / g_{i}}\right)+o g(f(n)) .
$$

Here we used that $N_{A}=N_{A^{\prime}}=\prod N_{A_{i}}^{e_{i}}$ and $N_{A_{i}}=N_{i}^{g_{i}}$, which imply $N_{A}=\prod N_{i}^{e_{i} g_{i}}$ and the square-free number $N_{i}$ divides $n=\operatorname{rad}\left(N_{A}\right)$. Then (6.10) proves (i).

We now show (ii). At the end of the proof of Theorem 5.1 (ii), we obtained in particular that $g \leq \log N_{A}$. Thus, the bound (ii) follows by replacing in the proof of (i) the estimate $v_{A^{\prime}} \leq \kappa n$ by the estimate $v_{A^{\prime}} \leq(8 g)^{6} \log N_{A}$, which is a consequence of d) obtained in course of the proof of Theorem 5.1 (ii). This completes the proof of Proposition 6.9.

Let $A$ be as in Proposition 6.9. Depending on the decomposition (in the $\mathbb{Q}$-isogeny category) of $A$ into $\mathbb{Q}$-simple abelian varieties $A \sim \prod A_{i}^{e_{i}}$, one can remove the factor $g$ in the main term of (6.9). For example, if all $e_{i}=1$ and if the $\mathbb{Q}$-simple factors $A_{i}$ of $A$ have pairwise coprime conductor, then (6.10) implies that (6.9) holds with $g f(n)$ replaced by $f(n)$. In general, one can always replace in (6.9) the quantity $g f(n)$ by $n \log \left(N_{A}\right) \log \log n$. Indeed, this follows directly from (6.10), because $\prod N_{i}^{e_{i}}$ divides $N_{A}=\prod N_{i}^{e_{i} g_{i}}$.

\section{Appendix B: A simplified version of Raynaud's bound for the variation of the Faltings height under isogenies}

In this appendix we simplify the shape of Raynaud's bound [54, Thm 4.4.9], which is based on Faltings' method [21] and refinements of Paršin and Zarhin.

We continue the notation of Section 3 and we denote by $h_{F}$ the stable Faltings height. Let $K$ be a number field of degree $d$ and discriminant $D_{K}$. Further, let $A$ be an abelian variety defined over $K$ of dimension $g \geq 1$, and let $\ell, \ell^{\prime}$ be two distinct rational primes that both do not divide the conductor $N_{A}$. Now, we can state the following. 
Proposition 6.10 ([54]). Suppose that $A$ is semistable. Then any abelian variety $A^{\prime}$ defined over $K$, which is $K$-isogenous to A, satisfies

$$
\left|h_{F}(A)-h_{F}\left(A^{\prime}\right)\right| \leq 8 g^{3} t\left(\ell^{2 m}+m \log \ell^{\prime}+4 g d^{2} \log D_{K}\right), \quad m=d g\left(\begin{array}{c}
2 g \\
g
\end{array}\right) .
$$

Here $t=\max (1,|T|)$ for $T$ the set of finite places of $K$ where $A$ has bad reduction.

We point out that one cannot replace $t$ by $|T|$ in (6.11), because $\left|h_{F}(A)-h_{F}\left(A^{\prime}\right)\right|=0$ is wrong for certain $A / K$ with $|T|=0$ as explained in (6.12). In particular, this shows that there is a minor inaccuracy in the explicit shape of Raynaud's original bound in [54, Thm 4.4.9], which we shall correct in Lemma 6.12.

\section{B.1 Proof of Proposition 6.10}

We continue our notation and we take $A, A^{\prime}$ as in Proposition 6.10. To prove the bound (6.11), we go into Raynaud's work [54] using his terminology. In particular, in what follows the numbering $x . y . z$ refers to $x . y . z$ in [54], isogeny means $K$-isogeny, $\mathcal{O}$ denotes the ring of integers of $K$ and $\bar{F}$ denotes an algebraic closure of a field $F$. Further, $T$ denotes the set of finite places of $K$ where $A$ has bad reduction and $g=\operatorname{dim}(A)$.

Let $p$ be a rational prime. We recall that the notion of a belle $p$-isogeny was introduced in Définition 4.1.1 and that the nonnegative rational integers $\Delta_{p}$ are defined in 4.4.7. The following more precise version of Corollaire 4.4 .8 will be used below.

Lemma 6.11 ([54]). Any p-isogeny $A \rightarrow A^{\prime}$ factors into a product

$$
\varphi_{1} \cdots \varphi_{k}, \quad k \leq \begin{cases}2 g & \text { if }|T|=0 \\ 4 g^{2}|T| & \text { if }|T| \geq 1\end{cases}
$$

of isogenies $\varphi_{i}$ such that $\operatorname{deg}\left(\varphi_{i}\right) \leq p^{4 g \Delta_{p}}$ or such that $\varphi_{i}$ factors into the product of a belle p-isogeny and two isogenies that both have degree at most $p^{2 g \Delta_{p}}$.

Proof. Lemme 4.4.3 factors any $p$-isogeny into a product $\varphi_{1} \cdots \varphi_{r}$ of isogenies $\varphi_{i}$ whose extensions $\varphi_{i}^{0}$ satisfy condition 1$) M_{i}(\bar{K}) \cong\left(\mathbb{Z} / p^{n_{i}} \mathbb{Z}\right)^{h_{i}}$ of 4.4 .7 , where $r \leq 2 g$ and $M_{i}$ is the kernel of the extension $\varphi_{i}^{0}$ of $\varphi_{i}$ to the open subschemes of the Néron models with connected fibres. In the case $|T| \geq 1$, Lemme 4.4.4 shows that after possibly further factoring each $\varphi_{i}$ into a product of at most $2 h_{i}|T| \leq 2 g|T|$ isogenies, we may and do assume that each $\varphi_{i}^{0}$ satisfies not only condition 1) but also condition 2) of 4.4.7: If $v \in T$, then $\left(\hat{M}_{i}\right)_{v}\left(\bar{K}_{v}\right)$ is a direct factor of $M_{i}\left(\bar{K}_{v}\right)$ where $K_{v}$ is the completion of $K$ at $v$ with ring of integers $\mathcal{O}_{v}$ and $\left(\hat{M}_{i}\right)_{v}$ is the finite part [54, (1.2)] of $M_{i} \otimes_{\mathcal{O}} \mathcal{O}_{v}$. In the case $|T|=0$, condition 2 ) of 4.4.7 is automatically satisfied because $T$ is empty.

We now show that each isogeny $\varphi_{i}$ has the desired properties. In the case when $n_{i}<2 \Delta_{p}$, we obtain that $\operatorname{deg}\left(\varphi_{i}\right)<p^{4 g \Delta_{p}}$ because $h_{i} \leq 2 g$. Suppose now that $n_{i} \geq 2 \Delta_{p}$. Then we may apply the arguments of 4.4.7 because $\varphi_{i}^{0}$ satisfies conditions 1) and 2). These arguments factor $\varphi_{i}$ into three isogenies: The middle isogeny is a belle $p$-isogeny and the extensions of the other two isogenies both satisfy condition 1) $M(\bar{K}) \cong\left(\mathbb{Z} / p^{n} \mathbb{Z}\right)^{h_{i}}$ of 4.4 .7 with $n=\Delta_{p}=n_{i}-\left(n_{i}-\Delta_{p}\right)$. Therefore, each of these other two isogenies has degree at most $p^{2 g \Delta_{p}}$ and then we conclude Lemma 6.11 .

We take two distinct rational primes $\ell$ and $\ell^{\prime}$ that both do not divide the conductor $N_{A}$. Then $A$ has good reduction at each finite place of $K$ dividing $\ell$ or $\ell^{\prime}$. Recall that for each rational prime $p \neq \ell$ the nonnegative integer $n_{p}=n_{p}(\ell)$ is defined in [54, p. 227] with respect to $\ell$ and put $n_{\ell, \ell^{\prime}}=n_{\ell}\left(\ell^{\prime}\right)$. Now, we define

$$
a=\sum 2 \Delta_{p} \log p, \quad b=\sum n_{p} \log p, \quad c=\left(2 \Delta_{\ell}+n_{\ell, \ell^{\prime}}\right) \log \ell
$$

with both sums taken over all $p \neq \ell$ in $S \cup R \cup\{2\}$, where $S$ is the set of rational primes $p \neq \ell$ with $n_{p} \geq 1$ and $R$ is the set of rational primes that ramify in $K$. The following result, involving $t=\max (1,|T|)$, 
gives a more precise version (and corrects minor inaccuracies in the explicit shape) of the bound for the variation in Théorème 4.4.9.

Lemma 6.12 ([54]). Define $\alpha=4 g^{3} t(a+b+c)$. Then it holds that

$$
\left|h_{F}(A)-h_{F}\left(A^{\prime}\right)\right| \leq \begin{cases}\alpha / 2 g & \text { if }|T|=0 \\ \alpha & \text { if }|T| \geq 1\end{cases}
$$

Proof. We begin by noting that Raynaud works in [54] with a different normalisation of the metric involved in the definition of the stable Faltings height $h_{F}$. However, the logarithm of the normalisation factor cancels out in the difference $h_{F}(A)-h_{F}\left(A^{\prime}\right)$.

In the case $|T|=0$, the explicit shape of the bound in Théorème 4.4.9 is not correct; the mistake is not serious and lies in the application of Corollaire 4.4.8, which is done in the paragraph of the proof of Théorème 4.4.9 starting with ' $\mathrm{D}$ ' après 4.4.7'. However, on replacing this paragraph by the version of Corollaire 4.4.8 given in Lemma 6.11, Raynaud's arguments give the bound $\alpha / 2 g$ for $|T|=0$ as claimed in Lemma 6.12.

In the case $|T| \geq 1$, the arguments of Théorème 4.4.9 give the bound $\alpha$ with the following two caveats. Firstly, as far as we can see, a factor 2 was forgotten in the last step of the proof where one puts everything together; this factor 2 is now taken into account in our $\alpha$. Secondly, we were not able to verify the proof of Corollaire 4.4.8 nor how 4.4.7 is applied in the proof of Théorème 4.4.9; the problem is that the arguments in 4.4.7 can only be applied to $p$-isogenies whose extensions (to the connected Néron models) satisfy conditions 1) and 2) such that the integer $n$ in 1) is at least $2 \Delta_{p}$. However, on using instead the version of Corollaire 4.4.8 given in Lemma 6.11, the arguments of Théorème 4.4 .9 go through and give the bound $\alpha$ for $|T| \geq 1$ as claimed in Lemma 6.12.

We are now ready to prove the simplified bound in Proposition 6.10.

Proof of Proposition 6.10. Lemma 6.12 gives that $\left|h_{F}(A)-h_{F}\left(A^{\prime}\right)\right| \leq 4 g^{3} t(a+b+c)$ and we now explicitly estimate from above the three quantities $a, b$ and $c$.

We first bound $a$. Let $p$ be a rational prime and let $v$ be a finite place of $K$ with residue characteristic $p$. We denote by $e_{v}$ the ramification index of $v$. It holds that $v(n)=e_{v} \operatorname{ord}_{p}(n)$ for each $n \in \mathbb{Z}$ and the different ideal $\mathfrak{D}_{\mathcal{O} / \mathbb{Z}}$ satisfies $v\left(\mathfrak{D}_{\mathcal{O} / \mathbb{Z}}\right)<e_{v}+v\left(e_{v}\right)$ by Dedekind's different theorem [3, B.2.11]. Therefore, the number $\delta_{v}$ appearing in the definition of $\Delta_{p}$ in 4.4 .7 is bounded by $\delta_{v} \leq \frac{1}{p-1}+1+\operatorname{ord}_{p}\left(e_{v}\right)$, and hence we obtain that $\delta_{v} \leq 1+e_{v} \leq 2 d$ because $\operatorname{ord}_{p}\left(e_{v}\right) \leq e_{v}-1$ and $e_{v} \leq d$ where $d=[K: \mathbb{Q}]$. This leads to

$$
\Delta_{p} \leq \begin{cases}4 g d^{2} & \text { if } p \in R \cup\{2\} \\ 0 & \text { otherwise. }\end{cases}
$$

Then, on using that the set $R$ is the set of rational prime divisors of the discriminant $D_{K}$ of $K$ by Dedekind's discriminant theorem [3, B.2.12], we obtain

$$
a+2 \Delta_{\ell} \log \ell=\sum_{p \in R \cup\{2\}} 2 \Delta_{p} \log p \leq 8 g d^{2} \log D_{K}+\frac{1}{2} \ell^{2 m}, \quad m=d g\left(\begin{array}{c}
2 g \\
g
\end{array}\right) .
$$

Here we used that $8 g d^{2} \log 2 \leq \frac{1}{2} \ell^{2 m}$, which follows from $16 g d^{2}=2^{r} \leq \ell^{r} \leq \ell^{2 m}$, where $r=$ $4+\log \left(g d^{2}\right) / \log 2$ is bounded by $r \leq 2 g d\left(2^{g}\right)^{d} \leq 2 m$.

Next, we bound $b$ and $c-2 \Delta_{\ell} \log \ell$. We take again a rational prime $p$. An application of Corollaire 4.3.5 with $n_{p}$ for $p \in S$ and $n_{\ell, \ell^{\prime}}=n_{\ell}\left(\ell^{\prime}\right)$ shows for $p \neq \ell$ that

$$
n_{p} \log p \leq\left\{\begin{array}{ll}
2 m \log \ell & \text { if } p \in S, \\
0 & \text { otherwise, }
\end{array} \quad \text { and } c-2 \Delta_{\ell} \log \ell=n_{\ell, \ell^{\prime}} \log \ell \leq 2 m \log \ell^{\prime}\right.
$$


Here $m / g d=\left(\begin{array}{c}2 g \\ g\end{array}\right)^{d} \geq\left(\begin{array}{c}2 g \\ g\end{array}\right)^{n}$ for $n=\max \left(N, N^{\prime}\right)$ with $N$ and $N^{\prime}$ the number of finite places of $K$ of residue characteristic $\ell$ and $\ell^{\prime}$, respectively, and $n_{p}=0$ for $p \notin S \cup\{\ell\}$ because $S$ is by definition the set of all primes $p \neq \ell$ with $n_{p} \geq 1$. Now, we deduce

$$
b=\sum_{p \in S} n_{p} \log p \leq|S| 2 m \log \ell \leq \frac{3}{2} \ell^{2 m}
$$

because $|S| \leq \frac{3}{2} \ell^{2 m} /(2 m \log \ell)$. This bound for $|S|$ can be obtained as follows: Corollaire 4.3 .5 gives that any $p \in S$ satisfies $p \leq p^{n_{p}} \leq \ell^{2 m}$ because $n_{p} \geq 1$ for $p \in S$, and therefore $|S|$ is bounded by the number $\pi(x)$ of rational primes at most $x=\ell^{2 m}$ that satisfies $\pi(x) \leq \frac{3}{2} x / \log x$ by the explicit prime number theorem in [60, Cor 1].

Finally, on combining the above displayed bounds, we deduce that $a+b+c$ is at most $2 \ell^{2 m}+$ $2 m \log \ell^{\prime}+8 g d^{2} \log D_{K}$ and this implies Proposition 6.10.

\section{B.2 Comments about $\left|h_{F}(A)-h_{F}\left(A^{\prime}\right)\right|=0$}

We continue our notation and we take $A, A^{\prime}$ as in Proposition 6.10. There are results in [54, §4.3] proving $\left|h_{F}(A)-h_{F}\left(A^{\prime}\right)\right|=0$ if $A$ and $A^{\prime}$ satisfy certain (strong) extra assumptions. However, $\left|h_{F}(A)-h_{F}\left(A^{\prime}\right)\right|=0$ is wrong for certain $A / K$ with $|T|=0$. To give an example, let $E$ be an elliptic curve over a number field $k$ such that $\operatorname{End}\left(E_{\bar{k}}\right)=\mathbb{Z}$ and such that $E$ has everywhere good reduction. In particular, $E$ is semistable. Then for each rational prime $p$, an application of Ullmo-Szpiro [65, Thm 1.1. (3)] with $E$ and $n=p^{2}$ gives

$$
h_{F}\left(E^{\prime}\right)-h_{F}(E)=\log p\left(1-\frac{1}{p}\right)+O(1)
$$

where $E^{\prime}=E_{k(P)} / G$ for $G$ the group (viewed as a constant group scheme over $k(P)$ ) generated by a point $P \in E(\bar{k})$ of order $p^{2}$ and where $O(1)$ only depends on $E / k$. Thus, after choosing the rational prime $p$ large enough, we obtain

$$
\left|h_{F}(A)-h_{F}\left(A^{\prime}\right)\right|>0
$$

for $A=E_{k(P)}, A^{\prime}=E^{\prime}$ and $K=k(P)$, because the stable Faltings height $h_{F}$ is invariant under any finite field extension. Notice that the abelian variety $A^{\prime}$ is $K$-isogenous to $A$ and it holds that $|T|=0$ because $A=E_{k(P)}$ has everywhere good reduction.

Acknowledgements. The mathematical content of this article is fully contained in the second part of [61]: I copied the statements and proofs of the corresponding results in [61]. The results were partly obtained when I was a member (2011/12) at the IAS Princeton, supported by the NSF (DMS-0635607), and they were completed and written up when I was a PostDoc (2012/13) at the IHÉS supported by an EPDI fellowship. I am grateful to the IAS and IHÉS for providing excellent working conditions. Further, I thank Ariyan Javanpeykar, Arno Kret, Benjamin Matschke, Richard Taylor and Jack Thorne for useful and motivating discussions and the referees for their comments.

Conflict of Interest: None.

\section{References}

[1] A. Baker and G. Wüstholz, Logarithmic Forms and Diophantine Geometry, New Mathematical Monographs, Vol. 9 (Cambridge University Press, Cambridge, 2007).

[2] G. V. Bely̆̌, 'Galois extensions of a maximal cyclotomic field', Izv. Akad. Nauk SSSR Ser. Mat. 43(2) (1979), 267-276, 479.

[3] E. Bombieri and W. Gubler, Heights in Diophantine Geometry, New Mathematical Monographs, Vol. 4 (Cambridge University Press, Cambridge, 2006).

[4] S. Bosch, W. Lütkebohmert and M. Raynaud, 'Néron models', in Ergebnisse der Mathematik und ihrer Grenzgebiete (3) [Results in Mathematics and Related Areas (3)], Vol. 21 (Springer, Berlin, 1990).

[5] J.-B. Bost, 'Arakelov geometry of abelian varieties', in Conference on Arithmetical Geometry, Vol. $96-51$ (Max Planck Institut für Mathematik, Bonn, Germany, 1996), 1-6. 
[6] J.-B. Bost, 'Périodes et isogenies des variétés abéliennes sur les corps de nombres (d'après D. Masser et G. Wüstholz)' [Periods and isogenies of abelian varieties over number fields], Astérisque 237(795) (1996), 115-161.

[7] C. Breuil, B. Conrad, F. Diamond and R. Taylor, 'On the modularity of elliptic curves over $\boldsymbol{Q}$ : wild 3-adic exercises', J. Amer. Math. Soc. 14(4) (2001), 843-939.

[8] A. Brumer and K. Kramer, 'The conductor of an abelian variety', Compos. Math. 92(2) (1994), 227-248.

[9] A. Brumer and J. H. Silverman, The number of elliptic curves over $\boldsymbol{Q}$ with conductor N, Manuscripta Math. 91(1) (1996), 95-102.

[10] H. Carayol, 'Sur les représentations $l$-adiques associées aux formes modulaires de Hilbert' [On 1-adic representations associated with Hilbert modular forms], Ann. Sci. École Norm. Sup. (4) 19(3) (1986), 409-468.

[11] J. Coates, 'An effective $p$-adic analogue of a theorem of Thue. III. The diophantine equation $y^{2}=x^{3}+k$ ', Acta Arith. 16 (1969/1970), 425-435.

[12] R. F. Coleman and B. Edixhoven, 'On the semi-simplicity of the $U_{p}$-operator on modular forms', Math. Ann. 310(1) (1998), 119-127.

[13] J. E. Cremona, J.-C. Lario, J. Quer and K. Ribet (eds.), 'Modular curves and abelian varieties', in Progress in Mathematics, Vol. 224 (Birkhäuser, Basel, 2004) 1-292.

[14] P. Deligne, 'Représentations $l$-adiques' [1-adic representations], Astérisque 127 (1985), 249-255.

[15] P. Deligne, 'Un théorème de finitude pour la monodromie' [A finiteness theorem for monodromy], in Discrete Groups in Geometry and Analysis (New Haven, Conn., 1984) (Birkhäuser Boston, , 1987), 1-19.

[16] F. Diamond and J. Shurman, A First Course in Modular Forms, Graduate Texts in Mathematics, Vol. 228 (Springer, New York, 2005).

[17] B. Edixhoven and R. de Jong, Bounds for Arakelov Invariants of Modular Curves, Computational Aspects of Modular Forms and Galois Representations (Princeton University Press, Princeton, NJ, 2011), 217-256.

[18] J. S. Ellenberg and A. Venkatesh, 'Reflection principles and bounds for class group torsion', Int. Math. Res. Not. IMRN 1 (2007), Art. ID rnm002.

[19] J. H. Evertse and K. Gyôry, Discriminant Equations in Diophantine Number Theory, New Mathematical Monographs (Cambridge University Press, Cambridge, 2016).

[20] G. Faltings, 'Arakelov's theorem for abelian varieties', Invent. Math. 73(3) (1983), 337-347.

[21] G. Faltings, 'Endlichkeitssätze für abelsche Varietäten über Zahlkörpern' [Finiteness theorems for abelian varieties over number fields], Invent. Math. 73(3) (1983), 349-366.

[22] G. Frey, 'Links between solutions of $A-B=C$ and elliptic curves', Number Theory (Ulm, 1987), Lecture Notes in Math., Vol. 1380 (Springer, New York, 1989), 31-62.

[23] G. Frey, 'On ternary equations of Fermat type and relations with elliptic curves', in Modular Forms and Fermat's Last Theorem (Boston, MA, 1995) (Springer, New York, 1997), 527-548.

[24] C. Fuchs, R. von Känel and G. Wüstholz, 'An effective Shafarevich theorem for elliptic curves', Acta Arith. 148(2) (2011), 189-203.

[25] É. Gaudron and G. Rémond, 'Polarisations et isogénies' [Polarizations and isogenies], Duke Math. J. 163(11) (2014), 20572108.

[26] É. Gaudron and G. Rémond, 'Théorème des périodes et degrés minimaux d'isogénies' [The period theorem and minimal degrees of isogenies], Comment. Math. Helv. 89(2) (2014), 343-403.

[27] É. Gaudron and G. Rémond, 'Nouveaux théorèmes d'isogénie' [New isogeny theorems], Preprint, 2020.

[28] G. van der Geer, 'Hilbert modular surfaces', Ergebnisse der Mathematik und ihrer Grenzgebiete (3) [Results in Mathematics and Related Areas (3)], Vol. 16 (Springer, Berlin, 1988).

[29] A. Grothendieck and M. Raynaud, 'Modéles de Néron et monodromie' [Neron models and monodromy], in Groupes de monodromie en Géometrie Algebrique, I, II, Lecture Notes in Math., Vol. 288 (Springer, Berlin, Heidelberg 1972), 313 -523.

[30] H. A. Helfgott and A. Venkatesh, 'Integral points on elliptic curves and 3-torsion in class groups', J. Amer. Math. Soc. 19(3) (2006), 527-550.

[31] A. Javanpeykar, 'Polynomial bounds for Arakelov invariants of Belyi curves', Algebra Number Theory 8(1) (2014), 89-140. With an appendix by Peter Bruin.

[32] R. de Jong and G. Rémond, 'Conjecture de Shafarevitch effective pour les revêtements cycliques' [Effective Shafarevich conjecture for cyclic coverings], Algebra Number Theory 5(8) (2011), 1133-1143.

[33] J. Jorgenson and J. Kramer, 'Bounds on Faltings's delta function through covers', Ann. Math. (2) 170(1) (2009), $1-43$.

[34] J. Jorgenson and J. Kramer, 'Effective bounds for Faltings's delta function', Ann. Fac. Sci. Toulouse Math. (6) 23(3) (2014), 665-698.

[35] R. von Känel, 'An effective proof of the hyperelliptic Shafarevich conjecture and applications', Thesis, ETH Zurich (2011).

[36] R. von Känel, 'Modularity and integral points on moduli schemes', 2013, arXiv:1310.7263.

[37] R. von Känel, 'An effective proof of the hyperelliptic Shafarevich conjecture', J. Théor. Nombres Bordeaux 26(1) (2014), 507-530.

[38] R. von Känel, 'Integral points on moduli schemes of elliptic curves', Trans. London Math. Soc. 1(1) (2014), 85-115.

[39] R. von Känel, 'On Szpiro's discriminant conjecture', Int. Math. Res. Not. IMRN 16 (2014), 4457-4491.

[40] R. von Känel and A. Kret, 'Integral points on Hilbert moduli schemes’, Preprint, 2019, arXiv:1904.03503. 
[41] R. von Känel and A. Kret, 'Integral points on coarse Hilbert moduli schemes', In preparation.

[42] R. von Känel and B. Matschke, 'Solving S-unit, Mordell, Thue, Thue-Mahler and generalized Ramanujan-Nagell equations via Shimura-Taniyama conjecture', Preprint, 2016, arXiv:1605.06079.

[43] C. Khare and J.-P. Wintenberger, 'Serre's modularity conjecture', Invent. Math. 178(3) (2009), 485-586.

[44] A. Levin, 'Siegel's theorem and the Shafarevich conjecture', J. Théor. Nombres Bordeaux 24(3) (2012), 705-727.

[45] P. Lockhart, M. Rosen and J. H. Silverman, 'An upper bound for the conductor of an abelian variety', J. Algebraic Geom. 2(4) (1993), 569-601.

[46] D. W. Masser and G. Wüstholz, 'Isogeny estimates for abelian varieties, and finiteness theorems', Ann. Math. (2) 137(3) (1993), 459-472.

[47] D. W. Masser and G. Wüstholz, 'Factorization estimates for abelian varieties', Inst. Hautes Études Sci. Publ. Math. 81 (1995), 5-24.

[48] H. Mayer, 'Self-intersection of the relative dualizing sheaf on modular curves $X_{1}(N)$ ', J. Théor. Nombres Bordeaux 26(1) (2014), 111-161.

[49] J. S. Milne, 'On the arithmetic of abelian varieties', Invent. Math. 17 (1972), 177-190.

[50] M. R. Murty and H. Pasten, 'Modular forms and effective Diophantine approximation', J. Number Theory 133(11) (2013), 3739-3754.

[51] A. N. Paršin, 'Algebraic curves over function fields. I', Izv. Akad. Nauk SSSR Ser. Mat. 32 (1968), 1191-1219.

[52] A. N. Paršin, 'Minimal models of curves of genus 2, and homomorphisms of abelian varieties defined over a field of finite characteristic', Izv. Akad. Nauk SSSR Ser. Mat. 36(1) (1972), 67-109.

[53] D. Poulakis, 'Corrigendum to the paper: "The number of solutions of the Mordell equation" [Acta. Arith. 88 (1999), no. 173-179]', Acta Arith. 92(4) (2000), 387-388.

[54] M. Raynaud, 'Hauteurs et isogénies’ [Heights and isogenies], Astérisque 127 (1985), 199-234.

[55] G. Rémond, 'Hauteurs thêta et construction de Kodaira' [Theta heights and Kodaira construction], J. Number Theory 78(2) (1999), 287-311.

[56] G. Rémond, 'Propriétés de la hauteur de Faltings' [Properties of the Faltings height], Preprint, 2019.

[57] K. A. Ribet, 'Galois action on division points of Abelian varieties with real multiplications', Amer. J. Math. 98(3) (1976), 751-804.

[58] K. A. Ribet, 'Twists of modular forms and endomorphisms of abelian varieties', Math. Ann. 253(1) (1980), 43-62.

[59] K. A. Ribet, 'Abelian varieties over $Q$ and modular forms', in Algebra and Topology (Korea Adv. Inst. Sci. Tech., Taejŏn, Korean, 1992), 53-79.

[60] J. B. Rosser and L. Schoenfeld, 'Approximate formulas for some functions of prime numbers', Illinois J. Math. 6(1) (1962), 64-94.

[61] J.-P. Serre, 'Facteurs locaux des fonctions zêta des variétes algébriques' [Local factors of zeta functions of algebraic varieties (definitions and conjectures)], in Séminaire DPP, Vol. 19 Secrétariat Math., Paris, (1969-1970).

[62] J.-P. Serre, 'Sur les représentations modulaires de degré 2 de Gal $(\bar{Q} / Q)$ ' [On modular representations of degree 2 of Gal $(\bar{Q} / Q)]$, Duke Math. J. 54(1) (1987), 179-230.

[63] J.-P. Serre and J. Tate, 'Good reduction of abelian varieties', Ann. Math. (2) 88 (1968), 492-517.

[64] G. Shimura, 'Introduction to the arithmetic theory of automorphic functions', in Kanô Memorial Lectures, Publications of the Mathematical Society of Japan, No. 11 (Iwanami Shoten, Tokyo, 1971).

[65] L. Szpiro and E. Ullmo, 'Variation de la hauteur de Faltings dans une classe de $\bar{Q}$-isogénie de courbe elliptique' [Variation of the Faltings height in a $\bar{Q}$-isogeny class of elliptic curves], Duke Math. J. 97(1) (1999), 81-97.

[66] R. Taylor and A. Wiles, 'Ring-theoretic properties of certain Hecke algebras', Ann. Math. (2) 141(3) (1995), $553-572$.

[67] E. Ullmo, 'Hauteur de Faltings de quotients de $J_{0}(N)$, discriminants d'algèbres de Hecke et congruences entre formes modulaires' [Faltings height of quotients of $J_{0}(N)$, Hecke algebra discriminants and congruences between modular forms], Amer. J. Math. 122(1) (2000), 83-115.

[68] A. Wiles, 'Modular elliptic curves and Fermat's last theorem', Ann. Math. (2) 141(3) (1995), 443-551. 